\title{
Geometric Integration of Hamiltonian Systems Perturbed by Rayleigh Damping
}

\author{
Klas MODIN ${ }^{a}$ and Gustaf SÖDERLIND ${ }^{b}$ \\ ${ }^{a}$ School of Engineering and Advanced Technology, Massey University \\ Private Bag 11 222, Palmerston North 4442, New Zealand \\ E-mail: k.e.f.modin@massey.ac.nz \\ ${ }^{b}$ Centre for Mathematical Sciences, Lund University \\ Box 118, SE-221 00 Lund, Sweden \\ E-mail: gustaf.soderlind@na.lu.se
}

September 27, 2018

\begin{abstract}
Explicit and semi-explicit geometric integration schemes for dissipative perturbations of Hamiltonian systems are analyzed. The dissipation is characterized by a small parameter $\varepsilon$, and the schemes under study preserve the symplectic structure in the case $\varepsilon=0$. In the case $0<\varepsilon \ll 1$ the energy dissipation rate is shown to be asymptotically correct by backward error analysis. Theoretical results on monotone decrease of the modified Hamiltonian function for small enough step sizes are given. Further, an analysis proving near conservation of relative equilibria for small enough step sizes is conducted.

Numerical examples, verifying the analyses, are given for a planar pendulum and an elastic 3-D pendulum. The results are superior in comparison with a conventional explicit Runge-Kutta method of the same order.
\end{abstract}

Key-words: Geometric numerical integration splitting methods weakly dissipative systems

2000 MSC: 65P10 37M15 70F40 


\section{Contents}

1 Introduction 2

2 Problem Description 4

3 Numerical Integration Schemes 6

4 Backward Error Analysis 9

4.1 Review of Framework . . . . . . . . . . . . . 9

4.2 Application to Perturbed Hamiltonian Problems . . . . . . . . . 11

4.3 Numerical Example: 2-D Pendulum . . . . . . . . . . . . . 16

5 Symmetry and Conservation of Momentum 19

6 Near Conservation of Relative Equilibria 22

6.1 Relation to Symplectic Integration of Problems with Attracting Invariant Tori . . . . . . . . . . . . . . . . . . . . . . 24

6.2 Numerical Example: Elastic 3-D Pendulum . . . . . . . . . . . 25

7 Conclusions

\section{Introduction}

In this paper we analyze geometric numerical integration algorithms for weakly dissipative perturbations of Hamiltonian systems. The dissipation rate is governed by a small parameter $0 \leq \varepsilon \ll 1$, as in the simple example

$$
\begin{aligned}
\dot{\boldsymbol{q}} & =M^{-1} \boldsymbol{p} \\
\dot{\boldsymbol{p}} & =-V^{\prime}(\boldsymbol{q})-\varepsilon D \dot{\boldsymbol{q}}
\end{aligned}
$$

where $\boldsymbol{q}, \boldsymbol{p} \in \mathbb{R}^{n}, M$ is a symmetric positive definite matrix, and $D$ is a symmetric positive semi-definite matrix. At $\varepsilon=0$ the system is Hamiltonian with $H(\boldsymbol{q}, \boldsymbol{p})=\frac{1}{2} \boldsymbol{p}^{\top} M^{-1} \boldsymbol{p}+V(\boldsymbol{q})$, and for $\varepsilon>0$ it is dissipative. This type of dissipation is the simplest example of Rayleigh damping (the general case is presented in Section 2 below).

There are many applications. For example, in rolling bearing design, the objective is to minimize friction losses. The dynamics of such mechanical systems is often well described by this model. In numerical simulations, it is then of importance that energy losses are correct. This means that a geometric integrator (nearly) conserving energy at $\varepsilon=0$, and giving the (nearly) correct dissipation rate as a function of $\varepsilon>0$, is desirable. Moreover, if possible, explicit methods are preferred. However, well-known reversible explicit methods, such as the two-step explicit midpoint rule, typically lose their stability in the presence of dissipation. Furthermore, explicit symplectic methods for separable Hamiltonian systems (e.g. Lobatto IIIA-IIIB) become implicit if dissipation is added. This motivates a special study and analysis of explicit geometric integrators for systems with weak Rayleigh damping.

The paper will address this question by examining splitting methods that take advantage of the linearity of the dissipative perturbation. Three integration schemes are investigated. All of these schemes are explicit in the sense that 
no numerical root solving is required. The first two of the methods are exponential integrators (meaning they require the solution of a linear ODE). The third method is either fully explicit, or linearly implicit (meaning it requires the solution of a linear equation). In our numerical examples, the three methods under study turn out to yield practically identical results. Although the presented methods are all second order accurate, it is an easy extension to construct higher order methods by using higher order compositions. By backward error analysis we show that the methods have asymptotically correct dissipation behavior as the dissipation parameter $\varepsilon \rightarrow 0^{+}$. Moreover, we analyze the decay of the modified Hamiltonian function, and give theoretical results on monotonicity of its evolution for small enough step sizes. We also analyze conservation of momentum, and near conservation of relative equilibria, in the case when both the conservative and the dissipative parts are invariant under a symmetry.

The examined methods are invariant under choice of coordinates in the configuration space. Therefore, they are well defined on any smooth configuration manifold without reference to a specific choice of coordinates. Throughout this paper we utilize the differential geometric framework of Riemannian geometry to describe the systems of interest. Although an important consequence, the main reason for doing so is not to include systems on non-linear spaces (constrained systems). Rather, the presented framework and corresponding analysis become more transparent in a differential geometric setting. Also, the fact that the result are derived for abstract manifolds is essential for some of the analysis carried out in the paper. (For example, independence of configuration coordinates is a key ingredient in our proof of preservation of symmetry invariance.) Moreover, well known results in differential geometry, in particular on structural stability, symmetry groups and momentum maps, and relative equilibria, become more easily available in this setting. For the convenience of the reader, we sometimes carry out invariant calculations even in cases when they are evident from a differential geometric point of view.

The idea of applying geometric integration schemes to weakly dissipative problems is certainly not new. For instance, it is well known that symplectic methods applied to perturbed integrable systems (e.g. the Van der Pols equation) are superior to standard methods, in the sense that weakly attractive invariant tori are much better preserved; see [8, Chap. XII]. (Although the concepts of relative equilibria and invariant tori are closely related, the latter can be seen as a special case of the former, the settings and analyses in this paper and in [8, Chap. XII] are not overlapping. A comparison between the two settings is given in Section 6.1 below.) Furthermore, in the paper 12 it is observed numerically that variational methods, in particular a semi-explicit version of the Newmark method, applied to weakly dissipative systems give much more accurate energy dissipation rates than standard methods. In the simplified case of conformal Hamiltonian systems, corresponding to $D=\mathrm{Id}$ in equation (1) above, it is possible to construct integrators that exactly preserve the corresponding geometric structure; see [18, 20. Concerning preservation of relative equilibria and numerical integration, there has been extensive work within the framework of energy-momentum methods; see [5, 4, 2, 3. Under suitable conditions, energy-momentum methods exactly preserve relative equilibria. However, energy momentum schemes are fully implicit. Also related to our work is the recent paper [14, which uses a splitting technique, similar to the one used in this paper, for the integration of post-Newtonian equations, possibly with weak 
dissipation due to relativistic effects.

The paper is organized as follows. A problem description is given in Section 2 Numerical integration schemes are presented in Section 3 A backward error analysis of the methods is given in Section 4 . The result of the analysis is verified by a numerical example (planar pendulum) in Section 4.3. In Section 5 we show that the methods preserve symmetry invariance, and conserve corresponding momentum maps. In conjunction, Section 6 contains a study of conditions for near conservation of relative equilibria, verified by a numerical example (elastic pendulum) in Section 6.2. Finally, conclusions are given in Section 7

Throughout the paper we assume that the reader is familiar with basic differential geometry. In particular, the following geometric concepts are used without reference: Riemannian manifolds, tangent and co-tangent bundles, tensor fields, Lie derivative, symplectic maps, Hamiltonian vector fields, flow of a vector field, exponential map. For the convenience of the reader, other more advanced, but still fundamental, geometric concepts are introduced and defined as they are used. The introductory textbook on geometric mechanics by Marsden and Ratiu [17] is more than enough to cover all the differential geometric concepts in this paper.

We adopt the following notation. $\mathscr{Q}$ denotes a manifold, $T \mathscr{Q}$ and $T^{*} \mathscr{Q}$ its corresponding tangent and co-tangent bundle. Throughout the paper $q$ denotes an element in $\mathscr{Q}$. Furthermore, $p$ denotes an element in the co-tangent space $T_{q}^{*} \mathscr{Q}$. Thus, $p$ is always associated with $q$. The pairing between an element $p \in$ $T_{q}^{*} \mathscr{Q}$ and $\dot{q} \in T_{q} \mathscr{Q}$ is denoted $\langle p, \dot{q}\rangle$. We use $z$ for a point in $T^{*} \mathscr{Q}$ corresponding to $(q, p)$. The derivative of a function $\phi$ on $\mathscr{Q}$ at the point $q$ (also called the tangent map) is denoted $T_{q} \phi$. The set of smooth vector field on a manifold $\mathscr{P}$ is denoted $\mathfrak{X}(\mathscr{P})$, and the set of diffeomorphisms of $\mathscr{P}$ is denoted $\operatorname{Diff}(\mathscr{P})$. The flow of a vector field $X \in \mathfrak{X}(\mathscr{P})$ is $\exp (t X)$, where $t$ is the time parameter and exp : $\mathfrak{X}(\mathscr{P}) \rightarrow \operatorname{Diff}(\mathscr{P})$ is the exponential map. The Lie derivate along $X$ is denoted $£_{X}$. The space of smooth real-valued functions on $\mathscr{P}$ is denoted $\mathcal{F}(\mathscr{P})$. If $H \in \mathcal{F}\left(T^{*} \mathscr{Q}\right)$, then its corresponding canonically Hamiltonian vector field is denoted $X_{H}$. The canonical Poisson bracket between $H, G \in \mathcal{F}\left(T^{*} \mathscr{Q}\right)$ is denoted $\{H, G\}$. The space of type $(r, s)$ tensor fields over $\mathscr{Q}$ is denoted $\mathcal{T}_{s}^{r}(\mathscr{Q})$.

\section{Problem Description}

Let $(\mathscr{Q}, \mathrm{g})$ be a Riemannian manifold of dimension $n$, where $\mathrm{g} \in \mathcal{T}_{2}^{0}(\mathscr{Q})$ is a metric tensor, i.e., symmetric and positive definite. In our context $\mathscr{Q}$ is the configuration space of a conservative mechanical system with Lagrangian function of standard form $L(\dot{q}, q)=\frac{1}{2} \mathrm{~g}_{q}(\dot{q}, \dot{q})-V(q)$. Thus, the metric tensor determines the kinetic energy of the system.

We are interested in dissipative perturbations of such systems. To this end, let $\mathrm{d} \in \mathcal{T}_{2}^{0}(\mathscr{Q})$ be a symmetric positive semi-definite tensor field called dissipation tensor. We shall assume that the rank of $\mathrm{d}$ is constant, i.e., that it is a regular tensor. Further, the Rayleigh dissipation function is defined by $R(q, \dot{q})=\frac{1}{2} \mathrm{~d}_{q}(\dot{q}, \dot{q})$. We consider the following dissipative perturbation of the Euler-Lagrange equation

$$
\frac{\mathrm{d}}{\mathrm{d} t} \frac{\partial L}{\partial \dot{q}}-\frac{\partial L}{\partial q}=-\varepsilon \frac{\partial R}{\partial \dot{q}},
$$


where $\varepsilon \geq 0$ is the perturbation parameter.

From a structural, as well as a numerical, point of view it is often preferable to transform the Euler-Lagrange equation into a first order Hamiltonian system on the co-tangent bundle phase space $T^{*} \mathscr{Q}$. The metric tensor induces an isomorphism $\mathcal{M}(q): T_{q} \mathscr{Q} \rightarrow T_{q}^{*} \mathscr{Q}$ by the flat operator $\dot{q} \mapsto \dot{q}^{b}:=\mathrm{g}_{q}(\dot{q}, \cdot)$. Its inverse defines the sharp operator $p \mapsto p^{\sharp}:=\mathcal{M}(q)^{-1} p$. $\mathcal{M}$ is sometimes called the inertia operator. Since the kinetic energy descends from the metric tensor, the Legendre transformation is given by the flat operator, so the momentum variable is $p:=\partial L / \partial \dot{q}=\dot{q}^{b}$. The Hamiltonian function, describing the total energy of the system, is given by the sum of kinetic and potential energies

$$
H(q, p)=\underbrace{\frac{1}{2} \mathrm{~g}_{q}\left(p^{\sharp}, p^{\sharp}\right)}_{T(q, p)}+V(q) .
$$

Substituting variables, the perturbed Euler-Lagrange equation (2) transforms into a perturbed Hamiltonian system

$$
\begin{aligned}
& \dot{q}=p^{\sharp} \\
& \dot{p}=-\frac{\partial H}{\partial q}-\varepsilon \mathrm{d}_{q}\left(p^{\sharp}, \cdot\right),
\end{aligned}
$$

where we have used that $\partial H / \partial p=p^{\sharp}$, which follows since $\mathrm{g}_{q}\left(p^{\sharp}, p^{\sharp}\right)=\left\langle p, p^{\sharp}\right\rangle$.

Since the Hamiltonian part of equation (4) is perturbed by a non-Hamiltonian perturbation, total energy is not conserved. Indeed, the time evolution of the total energy along solution curves is given by

$$
\frac{\mathrm{d}}{\mathrm{d} t} H(q, p)=\frac{\partial H}{\partial q} \dot{q}+\frac{\partial H}{\partial p} \dot{p}=-\varepsilon \mathrm{d}_{q}\left(p^{\sharp}, p^{\sharp}\right)=-\varepsilon \mathrm{d}_{q}(\dot{q}, \dot{q}) \leq 0 .
$$

Thus, the rôle of the dissipation tensor is to specify the rate of energy decay at each point in phase space.

The form of system (4) is invariant under point transformations, as we will now derive. Indeed, let $\mathscr{Q}$ and $\mathscr{S}$ be diffeomorphic manifolds and $\phi: \mathscr{S} \rightarrow \mathscr{Q}$ a diffeomorphism. Consider now the change of variables $(s, r)=T^{*} \phi(q, p)$, where $T^{*} \phi: T^{*} \mathscr{Q} \rightarrow T^{*} \mathscr{S}$ is the co-tangent lift of $\phi$ (cf. Marsden and Ratiu 17, Sect. 6.3]). (In coordinates, $(\boldsymbol{s}, \boldsymbol{r}) \leftrightarrow(\boldsymbol{q}, \boldsymbol{p})$ is defined by the generating function of second kind $G_{2}(\boldsymbol{q}, \boldsymbol{r})=\phi(\boldsymbol{q}) \cdot \boldsymbol{r}$.) We know already from standard Hamiltonian theory that the governing equation for the Hamiltonian part of the vector field in the variables $(s, r)$ transforms into a new canonical Hamiltonian system for a new Hamiltonian function $K$ on $T^{*} \mathscr{S}$ given by

$$
\begin{aligned}
K(s, r) & =H\left(T^{*} \phi^{-1}(s, r)\right) \\
& =\left\langle\left(T_{s} \phi\right)^{-1 *} r, \mathcal{M}(\phi(s))^{-1}\left(T_{s} \phi\right)^{-1 *} r\right\rangle+V(\phi(s)) \\
& =\langle r, \underbrace{\left(T_{s} \phi\right)^{-1} \mathcal{M}(\phi(s))^{-1}\left(T_{s} \phi\right)^{-1 *} r}_{r^{\sharp}}\rangle+V(\phi(s)) \\
& =\left\langle r, r^{\sharp}\right\rangle+\left(\phi^{*} V\right)(s) \\
& =\left(\phi^{*} \mathrm{~g}\right)_{s}\left(r^{\sharp}, r^{\sharp}\right)+\left(\phi^{*} V\right)(s)
\end{aligned}
$$

where $\left(\phi^{*} \mathrm{~g}\right)_{s}(u, v)=\mathrm{g}_{\phi(s)}\left(T_{s} \phi u, T_{s} \phi v\right)$ and $\left(\phi^{*} V\right)(s)=V(\phi(s))$ is the pull-back of $g$ and $V$. Thus, in the new variables $(s, r)$ the Hamiltonian part of system (4) 
takes again the same form but with the pull-backed quantities $\phi^{*}$ g and $\phi^{*} V$. Next, the vector field over $T^{*} \mathscr{Q}$ corresponding to the dissipation is given by $\varepsilon Y(q, p)=\left(0,-\varepsilon \mathrm{d}_{q}\left(p^{\sharp}, \cdot\right)\right)=:\left(0,-\varepsilon \mathcal{D}(q) p^{\sharp}\right)$, where $\mathcal{D}(q): T_{q} \mathscr{Q} \rightarrow T_{q}^{*} \mathscr{Q}$. The corresponding vector field in the $(s, r)$ variables is the push-forward of $Y$ by $T^{*} \phi$, which is given by $\left(T^{*} \phi\right)_{*} Y:=T\left(T^{*} \phi\right) \circ X \circ T^{*} \phi^{-1}$. In matrix notation

$$
\begin{aligned}
\left(T^{*} \phi\right)_{*} Y(s, r) & =\left[\begin{array}{cc}
T_{s} \phi^{-1} & 0 \\
\frac{\partial}{\partial s}\left(T_{s} \phi\right)^{*} r & \left(T_{s} \phi\right)^{*}
\end{array}\right]\left[\begin{array}{c}
0 \\
\mathcal{D}(\phi(s))\left(T_{s} \phi\right)^{-1 *} r
\end{array}\right] \\
& =\left[\begin{array}{cc}
\left(T_{s} \phi\right)^{*} \mathcal{D}(\phi(s)) \mathcal{M}(\phi(s))^{-1}\left(T_{s} \phi\right)^{-1 *} r
\end{array}\right] \\
& =[\underbrace{\left(T_{s} \phi\right)^{*} \mathcal{D}(\phi(s)) T_{s} \phi}_{\left.r^{\sharp} \mathrm{d}\right)_{s}(\cdot, \cdot)} \underbrace{\left(T_{s} \phi\right)^{-1} \mathcal{M}(\phi(s))^{-1}\left(T_{s} \phi\right)^{-1 *} r} \\
& =\left(0,\left(\phi^{*} \mathrm{~d}\right)_{s}\left(r^{\sharp}, \cdot\right)\right)
\end{aligned}
$$

In summary, this means that system (4) is determined by the three geometric objects (tensors) $\mathrm{g}, \mathrm{d}$ and $V$ in such a way that a change of variables $(s, r)=$ $T^{*} \phi(q, p)$ takes system (4) into a new system of the same form defined by the pulled back triple $\phi^{*} \mathrm{~g}, \phi^{*} \mathrm{~d}$ and $\phi^{*} V$. In other words, the following diagram commutes:

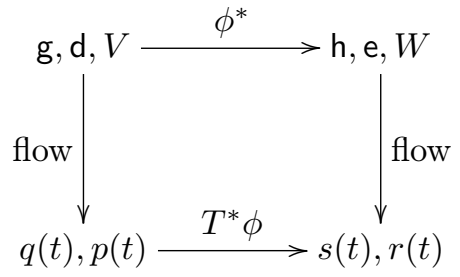

Thus, the form of equation (4) is independent of the choice of coordinates on $\mathscr{Q}$. The form is not, however, invariant with respect to any symplectic coordinate transformation on the full phase space $T^{*} \mathscr{Q}$ : only point transformations are allowed. As we will see, independence of coordinates on $\mathscr{Q}$ is preserved by the numerical schemes suggested in Section 3.

\section{$3 \quad$ Numerical Integration Schemes}

In this section we present the explicit and semi-explicit time-stepping schemes for the system (4) above to be analyzed in the paper. To begin with, we rewrite equation (4) in the more compact form

$$
\dot{z}=X_{T}(z)+X_{V}(z)+\varepsilon Y(z), \quad z=(q, p) \in T^{*} \mathscr{Q},
$$

where $X_{T}, X_{V}$ are the Hamiltonian vector fields corresponding to $T$ and $V$ respectively, and $\varepsilon Y$ is the non-Hamiltonian Rayleigh dissipation part.

Since the potential energy function $V$ is independent of $p$, the flow $\exp \left(t X_{V}\right)$ of $X_{V}$ is explicitly integrable for any choice of coordinates on $\mathscr{Q}$ simply by applying the forward Euler method. Throughout the rest of this paper we make one assumption which is crucial for the implementation of the suggested methods: that we know coordinates on $\mathscr{Q}$ in which the flow $\exp \left(t X_{T}\right)$ is explicitly 
computable. For particle systems, this is typically accomplished by choosing Cartesian coordinates in which the inertia operator $\mathcal{M}$ is independent of $q$. In this case, $\exp \left(t X_{T}\right)$ is again computable using the forward Euler method.

We study the following schemes:

Algorithm 3.1 (Three-term splitting method). Let $h>0$ be the step size and define $z_{k} \mapsto z_{k+1}$ by the map

$$
\Phi_{3 \mathrm{~S}}^{h}=\exp \left(\frac{h}{2} \varepsilon Y\right) \circ \exp \left(\frac{h}{2} X_{T}\right) \circ \exp \left(h X_{V}\right) \circ \exp \left(\frac{h}{2} X_{T}\right) \circ \exp \left(\frac{h}{2} \varepsilon Y\right) .
$$

Algorithm 3.2 (Two-term splitting method). Let $h>0$ be the step size and define $z_{k} \mapsto z_{k+1}$ by the map

$$
\Phi_{2 \mathrm{~S}}^{h}=\exp \left(\frac{h}{2} X_{T}\right) \circ \exp \left(h\left(X_{V}+\varepsilon Y\right)\right) \circ \exp \left(\frac{h}{2} X_{T}\right) .
$$

Algorithm 3.3 (Runge-Kutta splitting method). Let $h>0$ be the step size and let $\Psi_{X}^{h}$ be a Runge-Kutta method for $X \in \mathfrak{X}\left(T^{*} \mathscr{Q}\right)$. Define $z_{k} \mapsto z_{k+1}$ by the map

$$
\Phi_{\mathrm{RKS}}^{h}=\exp \left(\frac{h}{2} X_{T}\right) \circ \Psi_{X_{V}+\varepsilon Y}^{h} \circ \exp \left(\frac{h}{2} X_{T}\right) .
$$

All of these methods reduce to the classical Störmer-Verlet method $\Phi_{\mathrm{SV}}^{h}$ (also known as the leap-frog method or the Verlet scheme) in the case $\varepsilon=0$. (To be more precise, they reduce to the dual version, or $B$-version, of the classical Störmer-Verlet method; see [7] for various interpretations of this method.) Furthermore, Algorithm 3.1 and Algorithm 3.2 are second order accurate, as is Algorithm 3.3 if the Runge-Kutta method used is at least of order 2. It is straightforward to extend these methods to higher order, by using higher order compositions (see [19] for a review of splitting methods).

It should be noted that the vector field $X_{V}+\varepsilon Y$ is linear in $p$ and thus explicitly integrable using the exponential Euler method (cf. [10]). Hence, $\Phi_{2 S}^{h}$ is explicitly computable. Likewise, $Y$ is explicitly integrable using the exponential Euler method, so $\Phi_{3 \mathrm{~S}}^{h}$ is also explicitly computable. If the Runge-Kutta method in Algorithm 3.3 is explicit, then $\Phi_{\mathrm{RKS}}^{h}$ is fully explicit. If the Runge-Kutta method used is implicit, then each step of $\Phi_{\mathrm{RKS}}^{h}$ requires the solution of a linear system.

Runge-Kutta methods are well defined on linear spaces. Thus, one might think that Algorithm 3.3 is defined only in the case when $T^{*} \mathscr{Q} \simeq \mathbb{R}^{2 n}$. This is however not the case, since the dynamics of $X_{V}+\varepsilon Y$ is trivial in the configuration variable $q$, so each "Runge-Kutta step" $\Psi_{X_{V}+\varepsilon Y}^{h}$ takes place entirely on a co-tangent space $T_{q}^{*} \mathscr{Q}$, which is a linear space. Furthermore, since Runge-Kutta methods are invariant with respect to linear coordinate changes, and since any smooth change of configuration coordinates corresponds to a linear change of coordinates on each co-tangent space, Algorithm 3.3 is defined intrinsically (i.e., without reference to a specific choice of configuration coordinates).

Remark 3.1. Notice that system 8 is of the form

$$
\begin{aligned}
& \dot{q}=f(q, p) \\
& \dot{p}=g(q, p)
\end{aligned}
$$


so a partitioned Runge-Kutta method may be used to integrate it. The classical Störmer-Verlet method can be extended to the partitioned Runge-Kutta method given by the second order Lobatto IIIA-IIIB pair; see [8, Sect. II.2]. From our point of view, assuming that we are using a coordinate system in which the kinetic energy is independent of $q$, this extension of the classical StörmerVerlet method is conjugate to Algorithm 3.3 with the implicit midpoint rule as choice of Runge-Kutta method. Indeed, if $\Phi_{\mathrm{M}}^{h}, \Phi_{\mathrm{I}}^{h}$ and $\Phi_{\mathrm{E}}^{h}$ denotes, respectively, the implicit midpoint rule, the implicit Euler method and the explicit Euler method, it holds that $\Phi_{\mathrm{M}}^{h}=\Phi_{\mathrm{E}}^{h / 2} \circ \Phi_{\mathrm{I}}^{h / 2}$. Thus, Algorithm 3.3 with the implicit midpoint rule as the choice of Runge-Kutta method is $\exp \left(h X_{T} / 2\right) \circ \Phi_{\mathrm{E}}^{h / 2} \circ \Phi_{\mathrm{I}}^{h / 2} \circ$ $\exp \left(h X_{T} / 2\right)$. This method is conjugate to $\Phi_{\mathrm{I}}^{h / 2} \circ \exp \left(h X_{T}\right) \circ \Phi_{\mathrm{E}}^{h / 2}$, which is exactly the $A$-version of the partitioned Runge-Kutta extension of the classical Störmer-Verlet method.

As mentioned above, the suggested integrators are invariant with respect to choice of coordinates on the configuration space. From a differential geometric point of view, this result is obvious, since all the objects and operations used in their definition are intrinsic (configuration coordinate independent). However, for the convenience of the reader, we carry out a proof with complete calculations.

Proposition 3.1. Algorithms 3.1 3.3 are invariant with respect to choice of coordinates in $\mathscr{Q}$. That is, if $\mathscr{S} \simeq \mathscr{Q}$ and $\phi: \mathscr{S} \rightarrow \mathscr{Q}$ is a diffeomorphism, then the following diagram commute:

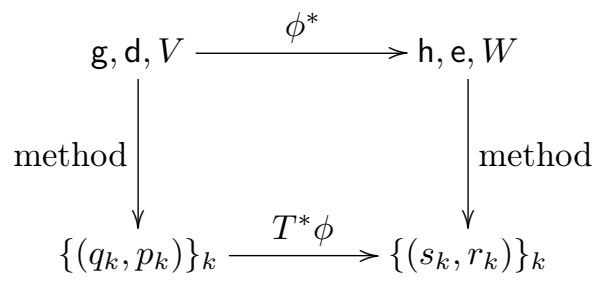

Proof. For simplicity we denote the map $T^{*} \phi: T^{*} \mathscr{Q} \rightarrow T^{*} \mathscr{S}$ by $\chi$. Further, we denote Algorithms 3.1 3.3 on $T^{*} \mathscr{Q}$ for the triple $(\mathrm{g}, \mathrm{d}, V)$ by $\Phi_{3 \mathrm{~S}}^{h}, \Phi_{2 \mathrm{~S}}^{h}$ and $\Phi_{\mathrm{RKS}}^{h}$. The corresponding algorithms on $T^{*} \mathscr{S}$ for the pulled back triple $(\mathrm{h}, \mathrm{e}, W):=$ $\left(\phi^{*} \mathrm{~g}, \phi^{*} \mathrm{~d}, \phi^{*} V\right)$ are denoted $\Upsilon_{3 \mathrm{~S}}^{h}, \Upsilon_{2 \mathrm{~S}}^{h}$ and $\Upsilon_{\mathrm{RKS}}^{h}$. We need to show that $\Phi_{3 \mathrm{~S}}^{h}=$ $\chi \circ \Upsilon_{3 \mathrm{~S}}^{h} \circ \chi^{-1}, \Phi_{2 \mathrm{~S}}^{h}=\chi \circ \Upsilon_{2 \mathrm{~S}}^{h} \circ \chi^{-1}$ and $\Phi_{\mathrm{RKS}}^{h}=\chi \circ \Upsilon_{\mathrm{RKS}}^{h} \circ \chi^{-1}$.

First, notice that the vector fields $X_{T}, \varepsilon Y$ and $X_{V}$ on $T^{*} \mathscr{Q}$ are defined respectively by the triples $(\mathrm{g}, 0,0),(0, \mathrm{~d}, 0)$ and $(0,0, V)$. Thus, from the commutative diagram (7) it follows that $\exp \left(t X_{U}\right)=\chi \circ \exp \left(t X_{T}\right) \circ \chi^{-1}, \exp (t Y)=$ $\chi \circ \exp (t Z) \circ \chi^{-1}$ and $\exp \left(t X_{V}\right)=\chi \circ \exp \left(t X_{W}\right) \circ \chi^{-1}$, where $X_{U}, \varepsilon Z$ and $X_{W}$ are the vector fields on $T^{*} \mathscr{S}$ defined respectively by the triples $(\mathrm{h}, 0,0)$, $(0, \mathrm{e}, 0)$ and $(0,0, W)$. From the definition of Algorithm 3.1 it now follows that $\Phi_{3 \mathrm{~S}}^{h}=\chi \circ \Upsilon_{3 \mathrm{~S}}^{h} \circ \chi^{-1}$.

Likewise, the vector field $X_{V}+\varepsilon Y$ is defined by the triple $(0, \mathrm{~d}, V)$. Thus, it follows again from diagram (7) that $\exp \left(t\left(X_{V}+\varepsilon Y\right)\right)=\chi \circ \exp \left(t\left(X_{W}+\varepsilon Z\right)\right) \circ \chi^{-1}$, and from the definition of Algorithm 3.2 we get $\Phi_{2 \mathrm{~S}}^{h}=\chi \circ \Upsilon_{2 \mathrm{~S}}^{h} \circ \chi^{-1}$.

Lastly, notice that $X_{V}+\varepsilon Y$ has trivial dynamics in $q$. Thus, it reduces to a system on $T_{q}^{*} Q$ (only the $p$ variable is affected by its flow). From diagram (7) it follows that $X_{W}+\varepsilon Z$ corresponds to a change of variables $q=\phi(s)$ and 
$r=T_{q}^{*} \phi \cdot p$. This is a linear map in the momentum variable. It follows that $\Psi_{X_{V}+\varepsilon Y}^{h}=\chi \circ \Psi_{X_{W}+\varepsilon Z}^{h} \circ \chi^{-1}$ since $\Psi^{h}$ is a linear method, i.e., invariant under a linear change of variables (every Runge-Kutta method is). It now follows that $\Phi_{\mathrm{RKS}}^{h}=\chi \circ \Upsilon_{\mathrm{RKS}}^{h} \circ \chi^{-1}$ from the definition of Algorithm 3.3 .

Remark 3.2. Although invariance with respect to choice of configuration coordinates for numerical integration of system (4) seems to be a highly natural requirement, most families of integrators do not have this property. For example, symplectic Runge-Kutta methods, which are invariant under a general linear change of coordinates and preserve the canonical symplectic form, are not invariant under co-tangent lifted point transformations. In engineering application, this has an impact, since the result of a numerical simulation then depends on whether Cartesian, cylindrical or spherical coordinates are used.

\section{Backward Error Analysis}

In this section we present a backward error analysis for the methods presented in Section 3. We start off with a brief review of the general notion of backward error analysis for ordinary differential equations. Thereafter, we show how the framework can be used for the class of Rayleigh damped problems considered in this paper.

\subsection{Review of Framework}

Let $\mathscr{P}$ be a phase space manifold and $X \in \mathfrak{X}(\mathscr{P})$ a smooth vector field. The set of diffeomorphisms on $\mathscr{P}$ is denoted $\operatorname{Diff}(\mathscr{P})$ (this set forms an infinite dimensional group under compositions). Following previous authors [23, 21, 9], we now define rigorously what is meant by an integrator.

Definition 4.1. An integrator for a vector field $X \in \mathfrak{X}(\mathscr{P})$ is a one-parameter family $\Phi^{h}: \mathscr{P} \rightarrow \mathscr{P}$ of diffeomorphisms that is smooth in $h$ and satisfies: $\Phi^{0}=\operatorname{Id}$ and $\Phi^{h}(z)-\exp (h X)(z)=\mathcal{O}\left(h^{r+1}\right)$ for all $z \in \mathscr{P}$, where $r \geq 1$ is the order of the integrator.

Given an integrator $\Phi^{h}$ for $X \in \mathfrak{X}(\mathscr{P})$, the basic notion of backward error analysis is to find a modified vector field $X_{h}$, depending smoothly on $h$, such that its flow coincides with $\Phi^{h}$, i.e., such that $\exp \left(h X_{h}\right)=\Phi^{h}$. Thus, the question of finding a modified vector field is equivalent to the question of finding an inverse of the exponential map exp : $\mathfrak{X}(\mathscr{P}) \rightarrow \operatorname{Diff}(\mathscr{P})$. However, it is well known that exp is not surjective, not even in an arbitrary small neighborhood of the identity (see e.g. 6]). Thus, consider instead the restriction to the real analytic case. Indeed, let $\mathscr{P}$ be a real analytic manifold (cf. [13), let $\mathfrak{X}^{a}(\mathscr{P}) \subset \mathfrak{X}(\mathscr{P})$ be the space of real analytic vector fields on $\mathscr{P}$, and let $\operatorname{Diff}^{a}(\mathscr{P}) \subset \operatorname{Diff}(\mathscr{P})$ be the corresponding real analytic diffeomorphisms. Even in this case, the restricted exponential map exp : $\mathfrak{X}^{a} \rightarrow \operatorname{Diff}^{a}(\mathscr{P})$ is not locally surjective [13, Chap. IX]. However, one can still find a formal modified vector field by asymptotic expansion in the step size parameter:

$$
X_{h}=X_{0}+h X_{1}+h^{2} X_{2}+\ldots
$$


where $X_{0}, X_{1}, X_{2}, \ldots \in \mathfrak{X}^{a}(\mathscr{P})$. Given an integrator $\Phi^{h}$ for $X \in \mathfrak{X}^{a}(\mathscr{P})$ the vector fields $X_{0}, X_{1}, X_{2}, \ldots$ are defined recursively by

$$
X_{k+1}(z)=\lim _{h \rightarrow 0} \frac{\Phi^{h}(z)-\exp \left(h X_{h, k}\right)(z)}{h^{k+1}}
$$

where $X_{0}=X$ and $X_{h, k}=\sum_{i=0}^{k} h^{i} X_{i}$. Thus, with this construction $\Phi^{h}(z)-$ $\exp \left(h X_{h, k}\right)(z)=\mathcal{O}\left(h^{k+1}\right)$, which follows from Taylor's theorem. Notice that if the integrator is of order $r$, then $X_{k}=0$ for $0<k<r$.

In general the formal series $\sqrt{9}$ does not converge, so instead one has to truncate the series. The basic result in backward error analysis states that there is an optimal truncation index $k$, depending on $h$, such that $\Phi^{h}-\exp \left(h X_{h, k}\right)$ is exponentially small. There are many contributors to this result: we refer to [8, Chap. IX] and references therein for an account of its origin. The original result is obtained for Euclidean phase space $\mathscr{P}=\mathbb{R}^{n}$. For the purpose of this paper, we use a generalization to manifolds given in [9]:

Theorem 4.1. Let $\mathscr{P}$ be a real analytic manifold, $\mathrm{U} \subset \mathscr{P}$ a compact subset, $X \in \mathfrak{X}^{a}(\mathscr{P})$ a real analytic vector field, and $\Phi^{h}$ an integrator for $X$ such that $h \mapsto \Phi^{h}(z)$ is real analytic for all $z \in \mathrm{U}$. Then there exists a distance function dist $: \mathscr{P} \times \mathscr{P} \rightarrow \mathbb{R}^{+}$, a truncation index $k$ (depending on $h$ ), and constants $C, \gamma, h_{0}>0$ such that

$$
\operatorname{dist}\left(\Phi^{h}(z), \exp \left(h X_{h, k}\right)(z)\right) \leq h C \mathrm{e}^{-\gamma / h}
$$

for all $z \in \mathrm{U}$ whenever $h \leq h_{0}$.

Remark 4.1. The distance function used in Theorem 4.1 is obtained by first embedding $\mathscr{P}$ in $\mathbb{R}^{n}$ (by the Whitney embedding theorem) and then restricting the Euclidean distance function on $\mathbb{R}^{n}$ to the embedded submanifold. In particular, in the case when $\mathscr{P}=\mathbb{R}^{n}$, the distance function used is just the usual Euclidean distance. See [9] for details.

Let us now consider the special case when $\mathscr{P}=T^{*} \mathscr{Q}$ (as in this paper). Denote by $\mathfrak{X}_{\text {Ham }}\left(T^{*} \mathscr{Q}\right)$ the space of Hamiltonian vector fields on $T^{*} \mathscr{Q}$ (with respect to the canonical symplectic form), i.e., $X \in \mathfrak{X}_{\mathrm{Ham}}\left(T^{*} \mathscr{Q}\right)$ implies that $X=X_{H}$ for some Hamiltonian function $H \in \mathcal{F}\left(T^{*} \mathscr{Q}\right)$. Correspondingly, we have the subgroup of exact symplectic diffeomorphisms $\operatorname{Diff}_{\mathrm{Ham}}\left(T^{*} \mathscr{Q}\right)$. It is well known that $\exp (X) \in \operatorname{Diff}_{\mathrm{Ham}}\left(T^{*} \mathscr{Q}\right)$ if and only if $X \in \mathfrak{X}_{\mathrm{Ham}}\left(T^{*} \mathscr{Q}\right)$.

Remark 4.2. Notice that there is, in general, a difference between Hamiltonian vector fields $\mathfrak{X}_{\mathrm{Ham}}\left(T^{*} \mathscr{Q}\right)$ and symplectic vector fields $\mathfrak{X}_{\mathrm{Sp}}\left(T^{*} \mathscr{Q}\right)$, the former being a subalgebra of the latter. Indeed, $X \in \mathfrak{X}_{\mathrm{Sp}}\left(T^{*} \mathscr{Q}\right)$ means that $X$ preserves the symplectic form, whereas $X \in \mathfrak{X}_{\mathrm{Ham}}\left(T^{*} \mathscr{Q}\right)$ means that there exists a globally defined $H \in \mathcal{F}\left(T^{*} \mathscr{Q}\right)$ such that $X=X_{H}$. Correspondingly, the group of exact symplectic maps $\operatorname{Diff}_{\mathrm{Ham}}\left(T^{*} \mathscr{Q}\right)$ is a subgroup of the group of symplectic maps $\operatorname{Diff}_{\mathrm{Sp}}\left(T^{*} \mathscr{Q}\right)$. See e.g. [13, 22] for further reading.

Now, let $\Phi^{h}$ be an integrator for $X_{H} \in \mathfrak{X}_{\text {Ham }}\left(T^{*} \mathscr{Q}\right)$. Naturally, $\Phi^{h}$ is called a symplectic integrator if $\Phi^{h} \in \operatorname{Diff}_{\mathrm{Sp}}\left(T^{*} \mathscr{Q}\right)$ for each fixed $h>0$, and it is called an exact symplectic integrator if $\Phi^{h} \in \operatorname{Diff}_{\mathrm{Ham}}\left(T^{*} \mathscr{Q}\right)$ for each fixed $h>0$. In addition to Theorem 4.1 another basic result in backward error analysis is that if 
$\Phi^{h}$ is an exact symplectic integrator, then each of the vector fields in the formal series $(9)$ belong to $\mathfrak{X}_{\mathrm{Ham}}\left(T^{*} \mathscr{Q}\right)$. Thus, each of these vector fields correspond to a Hamiltonian function. For a real analytic Hamiltonian system integrated by an exact symplectic integrator, application of Theorem 4.1 yields that if the numerical solution stays on a compact subset, then there exists a truncated modified Hamiltonian function (corresponding to a truncated modified Hamiltonian vector field) which is conserved by the numerical solution up to an exponentially small term for exponentially long times (see e.g. [8, Sect. IX.8]). Furthermore, it can be shown that the family of truncated modified Hamiltonian functions have a common Lipschitz constant, independent of the step size [8, Sect. IX.7.2].

A deeper consequence of the modified vector field preserving the Hamiltonian structure is that if $X_{H}$ is Arnold-Liouville integrable (cf. [8, Sect. X.1]), i.e., it has invariant tori, then an exact symplectic integrator will preserve perturbed tori for exponentially long times (under certain technical assumptions associated with KAM theory).

\subsection{Application to Perturbed Hamiltonian Problems}

In this section we derive the modified vector fields for the numerical methods suggested in Section 3. Our aim is to study the energy dissipation behavior of the methods, using a backward error analysis approach. The idea is to study the evolution in the numerical solution of a truncated modified Hamiltonian function. In particular, we show that up to an exponentially small term, the suggested methods have asymptotically correct energy behavior in the dissipation parameter $\varepsilon$. Furthermore, the evolution of a truncated modified Hamiltonian for Algorithm 3.1, is monotone up to an exponentially small term.

For convenience, we say that a numerical method for perturbed Hamiltonian systems of the form (4) is (exact) symplectic, if it is (exact) symplectic for $\varepsilon=0$. Thus, the suggested methods Algorithm 3.1 3.3 are exact symplectic, which follows directly from the splitting approach, and the fact that $\operatorname{Diff}_{\mathrm{Ham}}\left(T^{*} \mathscr{Q}\right)$ forms a group under composition. Throughout this section, we assume that phase space $T^{*} \mathscr{Q}$ carries a real analytic structure.

Let $Z_{\varepsilon}=X_{T}+X_{V}+\varepsilon Y$ be the vector field in equation 8 . From equation 5 it follows that

$$
H\left(\exp \left(t Z_{\varepsilon}\right)(z)\right)-H(z)=-\varepsilon \int_{0}^{t} \mathrm{~d}_{q(s)}\left(p(s)^{\sharp}, p(s)^{\sharp}\right) \mathrm{d} s
$$

where $(q(s), p(s))=\exp \left(s Z_{\varepsilon}\right)(z)$. Our aim is to find a modified analog of 10 corresponding a numerical integrator, ideally by replacing the Hamiltonian with a modified Hamiltonian, and the dissipation tensor with a modified dissipation tensor. In particular, there are two qualitative features of equation 10 that we would like to find analogs of in the numerical solution:

- $H\left(\exp \left(t Z_{\varepsilon}\right)(z)\right)-H(z)$ is proportional to $\varepsilon$;

- $H\left(\exp \left(t Z_{\varepsilon}\right)(z)\right)-H(z) \leq 0$ for $t \geq 0$.

We first give the general structure of the formal modified vector field corresponding to a symplectic method: 
Lemma 4.2. Let $\Phi^{h}$ be an exact symplectic integrator of order $r$ for problem (4). Then its formal modified vector field is of the form

$$
Z_{h, \varepsilon}=X_{T}+X_{V}+\varepsilon Y+\sum_{k=r}^{\infty} h^{k}\left(X_{H_{k}}+\varepsilon Y_{k, \varepsilon}\right)
$$

where $X_{H_{k}} \in \mathfrak{X}_{\mathrm{Ham}}\left(T^{*} \mathscr{Q}\right)$ and $Y_{k, \varepsilon} \in \mathfrak{X}\left(T^{*} \mathscr{Q}\right)$ depending smoothly on $\varepsilon$.

Proof. For $\varepsilon=0$ the formal series is of the form

$$
Z_{h, 0}=X_{T}+X_{V}+\sum_{k=r}^{\infty} h^{k} X_{H_{k}}
$$

since the method is exact symplectic. The result now follows from Taylor's theorem since $\Phi^{h}$ depends smoothly on $\varepsilon$.

From this result we get a modified analog of equation 10 for general exact symplectic integrators. Indeed, let $H_{h, N}=T+V+\sum_{k=r}^{N} h^{k} H_{k}$, with $H_{k}$ as in Lemma 4.2. Then we have the following result:

Theorem 4.3. Let $\Phi^{h}$ be an exact symplectic integrator of order $r$ for problem (4), and let $\mathrm{U} \subset T^{*} \mathscr{Q}$ be a compact subset. Assume that $Z_{\varepsilon}=X_{T}+X_{V}+\varepsilon Y$ is real analytic in $\mathrm{U}$, and that $h \mapsto \Phi^{h}(z)$ is real analytic for every $z \in \mathrm{U}$. Then there is a truncation index $N$ depending on $h$ and $\varepsilon$ such that

$$
\left|H_{h, N}\left(\Phi^{h}(z)\right)-H_{h, N}(z)+\varepsilon \int_{0}^{h} \mathrm{~d}_{h, N, \varepsilon}\left(\exp \left(s Z_{h, N, \varepsilon}\right)(z)\right) \mathrm{d} s\right| \leq h \lambda C \mathrm{e}^{-\gamma / h}
$$

whenever $h \leq h_{0}$ and $\varepsilon \leq \varepsilon_{0}$, where $\lambda, C, \gamma, h_{0}, \varepsilon_{0}>0$ are constants not depending on $h$ or $\varepsilon$, and where

$$
\mathrm{d}_{h, N, \varepsilon}(z):=\mathrm{d}_{q}\left(p^{\sharp}, p^{\sharp}\right)-\sum_{k=r}^{N} h^{k}\left\langle\mathrm{~d} H_{h, N}(z), Y_{k, \varepsilon}(z)\right\rangle,
$$

for $Y_{k, \varepsilon}$ as in Lemma 4.2.

Proof. From truncation of the modified vector field in Lemma 4.2 it follows that

$$
\begin{aligned}
£_{Z_{h, N, \varepsilon}} H_{h, N}(z) & =\left\langle\mathrm{d} H_{h, N}(z), Z_{h, N, \varepsilon}(z)\right\rangle \\
& =\left\langle\mathrm{d} H_{h, N}(z), X_{H_{h, N}(z)}+\varepsilon Y_{h, N, \varepsilon}(z)\right\rangle \\
& =\varepsilon\left\langle\mathrm{d} H_{h, N}(z), Y_{h, N, \varepsilon}(z)\right\rangle \\
& =-\varepsilon(\underbrace{\mathrm{d}_{q}\left(p^{\sharp}, p^{\sharp}\right)-\sum_{k=r}^{N} h^{k}\left\langle\mathrm{~d} H_{h, N}(z), Y_{k, \varepsilon}(z)\right\rangle}_{\mathrm{d}_{h, N, \varepsilon}(z)}) .
\end{aligned}
$$

Thus,

$$
H_{h, N}\left(\exp \left(t Z_{h, N, \varepsilon}\right)(z)\right)-H_{h, N}(z)=-\varepsilon \int_{0}^{t} \mathrm{~d}_{h, N, \varepsilon}\left(\exp \left(s Z_{h, N, \varepsilon}\right)(z)\right) \mathrm{d} s .
$$


Moreover, we have

$$
\begin{aligned}
H_{h, N}\left(\Phi^{h}(z)\right)-H_{h, N}(z) & =H_{h, N}\left(\exp \left(t Z_{h, N, \varepsilon}\right)(z)\right)-H_{h, N}(z) \\
& +H_{h, N}\left(\Phi^{h}(z)\right)-H_{h, N}\left(\exp \left(t Z_{h, N, \varepsilon}\right)(z)\right),
\end{aligned}
$$

so we get

$$
\begin{array}{r}
\left|H_{h, N}\left(\Phi^{h}(z)\right)-H_{h, N}(z)+\varepsilon \int_{0}^{t} \mathrm{~d}_{h, N, \varepsilon}\left(\exp \left(s Z_{h, N, \varepsilon}\right)(z)\right) \mathrm{d} s\right| \\
=\left|H_{h, N}\left(\Phi^{h}(z)\right)-H_{h, N}\left(\exp \left(t Z_{h, N, \varepsilon}\right)(z)\right)\right|
\end{array}
$$

Let $\lambda>0$ be an $h$-independent Lipschitz constant for $H_{h, N}$ with respect to the distance function dist in Theorem 4.1 (such a Lipschitz bound is known to exists for truncated modified Hamiltonians, see e.g. [8, Theorem 8.1, Sect. IX.8]). Thus, an estimate for the right hand side is

$$
\left|H_{h, N}\left(\Phi^{h}(z)\right)-H_{h, N}\left(\exp \left(n h Z_{h, N, \varepsilon}\right)(z)\right)\right| \leq \lambda \operatorname{dist}\left(\Phi^{h}(z), \exp \left(h Z_{h, N, \varepsilon}\right)(z)\right) .
$$

Now, in order to get a bound which is uniform in $\varepsilon$, let $\varepsilon_{0}>0$ and consider the extended map $\hat{\Phi}^{h}(z, \varepsilon):=\left(\Phi^{h}(z), \varepsilon\right)$ on $\mathrm{U} \times\left[0, \varepsilon_{0}\right]$ (notice that $\Phi^{h}$ depends on $\varepsilon$ ), which is an integrator for the extended vector field $\hat{Z}(z, \varepsilon):=\left(Z_{\varepsilon}(z), 0\right)$. Since the extended $\varepsilon$-part is integrated exactly by $\hat{\Phi}^{h}$, it holds that $\exp \left(h \hat{Z}_{h, N}\right)(z, \varepsilon)=$ $\left(\exp \left(h Z_{h, N, \varepsilon}\right)(z), \varepsilon\right)$, so we have

$$
\widehat{\operatorname{dist}}\left(\hat{\Phi}^{h}(z, \varepsilon), \exp \left(h \hat{Z}_{h, N}\right)(z, \varepsilon)\right)=\left(\operatorname{dist}\left(\Phi^{h}(z), \exp \left(h Z_{h, N, \varepsilon}\right)(z)\right)^{2}+|\varepsilon-\varepsilon|^{2}\right)^{\frac{1}{2}} .
$$

Thus, in combination with (11) we have

$$
\begin{aligned}
\left|H_{h, N}\left(\Phi^{h}(z)\right)-H_{h, N}\left(\exp \left(n h Z_{h, N, \varepsilon}\right)(z)\right)\right| & \leq \lambda \widehat{\operatorname{dist}}\left(\hat{\Phi}^{h}(z, \varepsilon), \exp \left(h \hat{Z}_{h, N}\right)(z, \varepsilon)\right) \\
& \leq h \lambda C \mathrm{e}^{-\gamma / h}
\end{aligned}
$$

for $h \leq h_{0}>0$, where the last estimate follows from Theorem 4.1 applied to the extended integrator $\hat{\Phi}^{h}$, which can be done since $\mathrm{U} \times\left[0, \varepsilon_{0}\right]$ is compact and $\hat{Z}(z, \varepsilon)$ is real analytic on $\mathrm{U} \times\left[0, \varepsilon_{0}\right]$. This proves the theorem.

From the result we see that, up to an exponentially small term, the modified energy evolves with a rate which is $\mathcal{O}\left(\varepsilon h^{r}\right)$ close to the energy dissipation rate of the exact solution. Thus, the dynamics is asymptotically correct in the perturbation parameter $\varepsilon$.

In transient regions of the phase space, i.e., regions where the value of dissipation tensor $\mathrm{d}$ is relatively large, Theorem 4.3 asserts that the energy evolves in a monotone fashion. More precisely, let $\mathrm{U} \subset T^{*} \mathscr{Q}$ be a compact subset such that $\sup _{z \in \mathrm{U}}\left|\mathrm{d}_{h, N, \varepsilon}(z)-\mathrm{d}_{q}\left(p^{\sharp}, p^{\sharp}\right)\right| / \mathrm{d}_{q}\left(p^{\sharp}, p^{\sharp}\right) \ll 1$ for the chosen step size. As long as the numerical solution stays in $\mathrm{U}$, Theorem 4.3 then asserts that the modified Hamiltonian is decreasing from step to step, and that the numerical dissipation rate is accurate relative to the exact dissipation rate. Thus, the transient phase in the simulation is captured very well with a symplectic integrator, which is often important in application, for example when accurate estimates of energy losses are essential. 
After the transient phase some type of "steady state" dynamics is commonly reached. In phase space, this often occur close to the subset ker $d=\{z \in$ $\left.T^{*} \mathscr{Q} ; \mathrm{d}_{q}\left(p^{\sharp}, p^{\sharp}\right)=0\right\}$, i.e., where the dissipation vanishes. Furthermore, it is common that dissipative forces (e.g. friction forces) are small relative to conservative forces. In such regions, one does not necessarily have that $d_{h, N, \varepsilon}(z) \geq 0$, so Theorem 4.3 does not assert that the evolution of the modified Hamiltonian is monotone. However, for the special case of the suggested Algorithm 3.1, we now derive a refined version of Theorem 4.3 , for which monotone decrease of the modified Hamiltonian is asserted for small enough step sizes (up to an exponentially small correction term). The result is based on the following two lemmas:

Lemma 4.4. The formal modified Hamiltonian $H_{h}$ for the Störmer-Verlet method $\Phi_{S V}^{h}$ applied to problem (8) with $\varepsilon=0$ is of the form

$$
\begin{aligned}
H_{h}(q, p)=\mathrm{g}_{q}\left(p^{\sharp}, p^{\sharp}\right)+V(q) & +\sum_{\ell=1}^{\infty} h^{2 \ell} V_{2 \ell}(q) \\
& +\sum_{k=1}^{\infty} \sum_{\ell=1}^{\infty} h^{2(k+\ell-1)}\left(\mathrm{g}_{2 k, 2 \ell}\right)_{q}(\underbrace{p^{\sharp}, \ldots, p^{\sharp}}_{2 k \text { copies }})
\end{aligned}
$$

where $V_{2 \ell} \in \mathcal{F}\left(T^{*} \mathscr{Q}\right)$ and $\mathrm{g}_{2 k, 2 \ell} \in \mathcal{T}_{2 k}^{0}(\mathscr{Q})$ are a totally symmetric tensor fields.

Proof. If $\mathrm{a} \in \mathcal{T}_{k}^{0}(\mathscr{Q})$ and $\mathrm{b} \in \mathcal{T}_{\ell}^{0}(\mathscr{Q})$ are two totally symmetric tensor fields, then the Poisson bracket between the functions $(q, p) \mapsto \mathrm{a}_{q}\left(p^{\sharp}, \ldots, p^{\sharp}\right)$ and $(q, p) \mapsto$ $\mathrm{b}_{q}\left(p^{\sharp}, \ldots, p^{\sharp}\right)$ is given by $(q, p) \mapsto \mathrm{c}_{q}\left(p^{\sharp}, \ldots, p^{\sharp}\right)$, where $\mathrm{c} \in \mathcal{T}_{k+\ell}^{0}(\mathscr{Q})$ is the totally symmetric tensor

$$
\begin{gathered}
\mathrm{c}_{q}(\cdot, \ldots, \cdot)=\mathrm{a}_{q}\left(\partial_{q} \mathrm{~b}_{q}(\cdot, \ldots, \cdot), \cdot, \ldots, \cdot\right)+\ldots+\mathrm{a}_{q}\left(\cdot, \ldots, \cdot, \partial_{q} \mathrm{~b}_{q}(\cdot, \ldots, \cdot)\right) \\
-\mathrm{b}_{q}\left(\partial_{q} \mathrm{a}_{q}(\cdot, \ldots, \cdot), \cdot, \ldots, \cdot\right)+\ldots+\mathrm{b}_{q}\left(\cdot, \ldots, \cdot, \partial_{q} \mathrm{a}_{q}(\cdot, \ldots, \cdot)\right) .
\end{gathered}
$$

Since Störmer-Verlet is a splitting method, and since $V$ and $g$ are tensors, it follows from the Baker-Campbell-Hausdorff formula that the modified Hamiltonian is a sum of terms of the form $h^{i+j} \mathrm{~g}_{i, j}\left(p^{\sharp}, \ldots, p^{\sharp}\right)$, where $\mathrm{g}_{i, j} \in \mathcal{T}_{i}^{0}(\mathscr{Q})$. Further, since the Störmer-Verlet method preserves reversibility it holds that $H_{h}(q, p)=H_{h}(q,-p)$. Thus, only the even order tensors remain. Also, only the even powers of $h$ survive, since the method is symmetric. This yields the result.

Lemma 4.5. Let $Y \in \mathfrak{X}\left(T^{*} \mathscr{Q}\right)$ be the dissipative vector field in equation (8), and let $H_{h, N}$ be the truncation of the formal modified Hamiltonian in Lemma 4.2. Further, let $\mathrm{U} \subset T^{*} \mathscr{Q}$ be a compact subset. Then there exists $h_{0}>0$ such that

$$
£_{Y} H_{h, N}=-\mathrm{d}_{q}\left(E_{h, N}(z) p^{\sharp}, p^{\sharp}\right) \leq 0
$$

for all $z \in \mathrm{U}$ whenever $h \leq h_{0}$, where $E_{h, N}(z): T_{q} \mathscr{Q} \rightarrow T_{q} \mathscr{Q}$ is an operator which is self-adjoint and positive definite with respect to $\mathrm{d}$, and such that $E_{h, N}(z)-\mathrm{Id}=\mathcal{O}\left(h^{2}\right)$ for all $z \in \mathrm{U}$.

Proof. Let $\langle\langle\cdot, \cdot\rangle\rangle$ denote the metric $\mathrm{g}_{q}(\cdot, \cdot)$. Let $C(q): T_{q} \mathscr{Q} \rightarrow T_{q} \mathscr{Q}$ be the positive semi-definite self-adjoint operator defined by $\langle\langle C(q) u, v\rangle\rangle=\mathrm{d}_{q}(u, v)$. In other words, $C(q) u=\mathrm{d}_{q}(u, \cdot)^{\sharp}$. 
It holds that

$$
\begin{aligned}
£_{Y}\left(\mathrm{~g}_{2 k, 2 \ell}\right)_{q}\left(p^{\sharp}, \ldots, p^{\sharp}\right)= & \left(\mathrm{g}_{2 k, 2 \ell}\right)_{q}\left(C(q) p^{\sharp}, p^{\sharp}, \ldots, p^{\sharp}\right)+ \\
& \left(\mathrm{g}_{2 k, 2 \ell}\right)_{q}\left(p^{\sharp}, C(q) p^{\sharp}, p^{\sharp}, \ldots, p^{\sharp}\right)+\ldots+ \\
& \left(\mathrm{g}_{2 k, 2 \ell}\right)_{q}\left(p^{\sharp}, \ldots, p^{\sharp}, C(q) p^{\sharp}\right)=:\left(\mathrm{d}_{2 k, 2 \ell}\right)_{q}\left(p^{\sharp}, \ldots, p^{\sharp}\right),
\end{aligned}
$$

which defines the totally symmetric tensor $\mathrm{d}_{2 k, 2 \ell} \in \mathcal{T}_{2 k}^{0}\left(T^{*} \mathscr{Q}\right)$. Notice that the kernel of $\mathrm{d}_{2 k, 2 \ell}$ is contained in the kernel of $C(q)$, i.e., if $u \in \operatorname{ker}(C(q))$, then $\left(\mathrm{d}_{2 k, 2 \ell}\right)_{q}(u, \cdot, \ldots, \cdot)=0$. Next, we define the self-adjoint operator $C_{2 k, 2 \ell}(z)$ : $T_{q} \mathscr{Q} \rightarrow T_{q} \mathscr{Q}$ by $\left\langle\left\langle C_{2 k, 2 \ell}(z) u, v\right\rangle\right\rangle=\left(\mathrm{d}_{2 k, 2 \ell}\right)_{q}\left(p^{\sharp}, \ldots, p^{\sharp}, u, v\right)$. Again, notice that $\operatorname{ker}\left(C_{2 k, 2 \ell}(z)\right) \subset \operatorname{ker}(C(q))$. All together, summing up the terms up to order $h^{N}$ in Lemma 4.4 , we get

$$
£_{Y} H_{h, N}(q, p)=-\left\langle\left\langle C(q) p^{\sharp}, p^{\sharp}\right\rangle\right\rangle-h^{2}\left\langle\left\langle C_{h, N}^{\Delta}(z) p^{\sharp}, p^{\sharp}\right\rangle\right\rangle
$$

where $C_{h, N}^{\Delta}(z)$ is a self-adjoint operator $T_{q} \mathscr{Q} \rightarrow T_{q} \mathscr{Q}$, with $\operatorname{ker}\left(C_{h, N}^{\Delta}(z)\right) \subset$ $\operatorname{ker}(C(q))$. Since both $C(q)$ and $C_{h, N}^{\Delta}(z)$ are self-adjoint it holds that $(\operatorname{ker} C(q))^{\perp}$ is an invariant subspace for both of them (follows from the spectral theorem). Since $d$ has constant rank and since $U$ is compact, it follows that

$$
\inf _{z \in \mathrm{U}}\left\|\left.C(q)\right|_{(\operatorname{ker} C(q))^{\perp}}\right\|^{2}>0 .
$$

Thus, there is an $h_{0}>0$ such that $C(q)+h^{2} C_{h, N}^{\Delta}(z)$ stays positive semi-definite when $h \leq h_{0}$ and $z \in \mathrm{U}$; the zero eigenvalues stays zero, and the perturbation, acting in $(\operatorname{ker} C(q))^{\perp}$, is small enough for its positive eigenvalues to stay positive. Hence,

$$
£_{Y} H_{h, N}(z)=-\left\langle\left\langle\left(C(q)+h^{2} C_{h, N}^{\Delta}(z)\right) p^{\sharp}, p^{\sharp}\right\rangle\right\rangle \leq 0
$$

if $h \leq h_{0}$ and $z \in \mathrm{U}$. Next, define $E_{h, N}(z): T_{q} \mathscr{Q} \rightarrow T_{q} \mathscr{Q}$ by $\mathrm{d}_{q}\left(E_{h, N}(z) u, v\right)=$ $\left\langle\left\langle\left(C(q)+h^{2} C_{h, N}^{\Delta}(z)\right) u, v\right\rangle\right\rangle$ for $u, v \in(\operatorname{ker} C(q))^{\perp}$ and $\left.E_{h, N}(z)\right|_{\operatorname{ker} C(q)}=$ Id.

We now give the refined result on evolution of the modified Hamiltonian function for Algorithm 3.1.

Theorem 4.6. Let $\mathrm{U} \subset T^{*} \mathscr{Q}$ be a compact subset, and assume that $\mathrm{g}, \mathrm{d}, V$ are real analytic on $\mathrm{U}$. Then, for the integrator $\Phi_{3 S}^{h}$, defined by Algorithm 3.1, there exists $h_{0}>0$, depending on $\mathrm{U}, \mathrm{g}, \mathrm{d}, V$ but not on $\varepsilon$, and a truncation index $N$ depending on $h$, such that

$$
\begin{aligned}
& \mid H_{h, N}\left(\Phi_{3 S}^{h}(z)\right)-H_{h, N}(z)+\varepsilon \int_{0}^{h / 2} \mathrm{~d}_{h, N}(\exp (s \varepsilon Y)(z)) \mathrm{d} s \\
& \quad+\varepsilon \int_{0}^{h / 2} \mathrm{~d}_{h, N}\left(\exp (s \varepsilon Y) \circ \Phi_{S V}^{h} \circ \exp (h \varepsilon Y / 2)(z)\right) \mathrm{d} s \mid \leq h \lambda C \mathrm{e}^{-\gamma / h}
\end{aligned}
$$

whenever $h \leq h_{0}$ and $z \in \mathrm{U}$, where $\lambda, C, \gamma, h_{0}>0$ are constants not depending on $\varepsilon$, and where

$$
\mathrm{d}_{h, N}(z):=\mathrm{d}_{q}\left(E_{h, N}(z) p^{\sharp}, p^{\sharp}\right) \geq 0
$$

with $E_{h, N}(z): T_{q} \mathscr{Q} \rightarrow T_{q} \mathscr{Q}$ as in Lemma 4.5. 


\section{Phase diagrams for damped pendulum using Algorithms 3.13 .3}
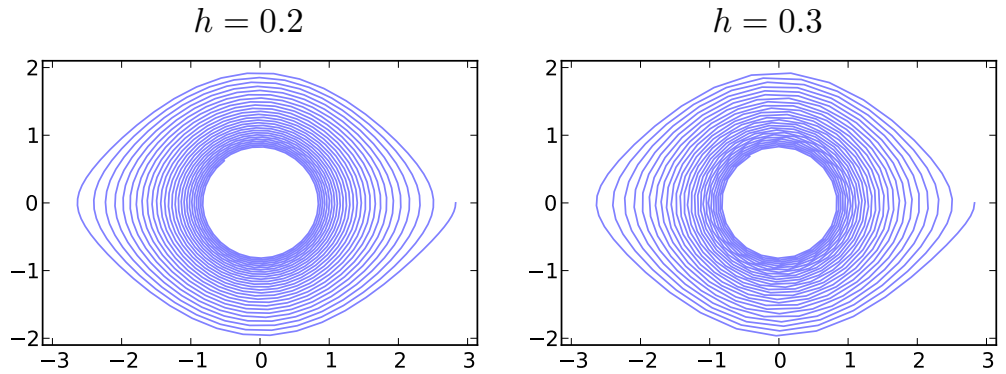

$$
h=0.4
$$
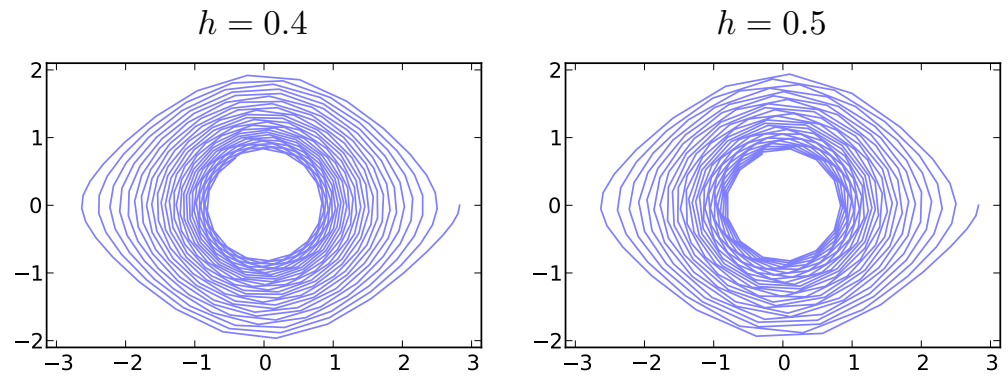

Figure 1: Phase diagrams for the damped pendulum problems integrated with the suggested methods (they all overlap at this level of detail) for various step sizes. Notice that the amount of dissipation does not depend much on the step size (the size of the hole in the middle is roughly the same).

Proof. We have

$$
\begin{aligned}
& H_{h, N}\left(\Phi_{3 \mathrm{~S}}^{h}(z)\right)-H_{h, N}(z)=H_{h, N}(\exp (h \varepsilon Y / 2)(z))-H_{h, N}(z) \\
& \quad+H_{h, N}\left(\Phi_{\mathrm{SV}}^{h} \circ \exp (h \varepsilon Y / 2)(z)\right)-H_{h, N}(\exp (h \varepsilon Y / 2)(z)) \\
& \quad+H_{h, N}\left(\exp (h \varepsilon Y / 2) \circ \Phi_{\mathrm{SV}}^{h} \circ \exp (h \varepsilon Y / 2)(z)\right)-H_{h, N}\left(\Phi_{\mathrm{SV}}^{h} \circ \exp (h \varepsilon Y / 2)(z)\right)
\end{aligned}
$$

From Theorem 4.3 with $\varepsilon=0$ it follows that

$$
H_{h, N}\left(\Phi_{\mathrm{SV}}^{h} \circ \exp (h \varepsilon Y / 2)(z)\right)-H_{h, N}(\exp (h \varepsilon Y / 2)(z)) \leq h \lambda C \mathrm{e}^{-\gamma / h}
$$

whenever $h \leq h_{0}^{\prime}$ for $\lambda, C, \gamma, h_{0}^{\prime}$ independent of $\varepsilon$ (since it is zero when we apply the theorem). Since $\exp (h \varepsilon Y / 2)$ is the exact $h$-flow of $\varepsilon Y / 2$ it follows from Lemma 4.5 that there exists $h_{0}^{\prime \prime}>0$ (clearly independent of $\varepsilon$ ) such that $\mathrm{d}_{h, N}(z)=\mathrm{d}_{q}\left(E_{h, N}(z) p^{\sharp}, p^{\sharp}\right) \geq 0$. This gives the four remaining terms in the sum. Now chose $h_{0}=\min \left(h_{0}^{\prime}, h_{0}^{\prime \prime}\right)$.

Notice in particular that this result asserts, up to an exponentially small rest term, that the modified Hamiltonian decreases monotonely for small enough step sizes. The result is optimal, in the sense that the same exponential term would remain if we take $\varepsilon=0$.

\subsection{Numerical Example: 2-D Pendulum}

Let $\mathscr{Q}=\mathbb{R}, \mathrm{g}_{q}(u, v)=\frac{1}{2} u v, \mathrm{~d}=\mathrm{g}$ and $V(q)=1-\cos (q)$. The system so obtained describes a damped non-linear pendulum in the vertical plane, with 
Phase diagrams for damped pendulum using Heun's method

$h=0.2$

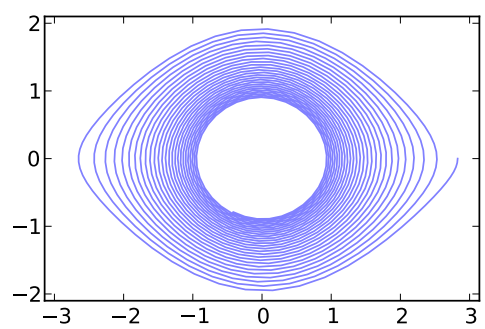

$h=0.4$

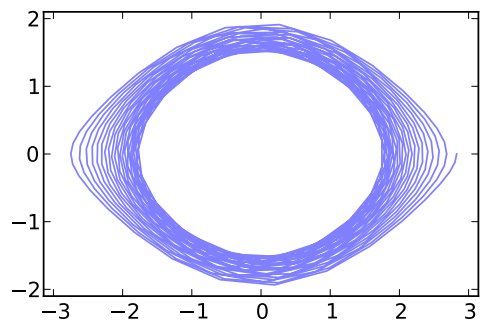

$h=0.3$

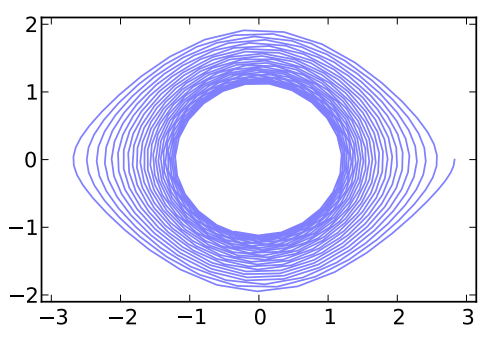

$h=0.5$

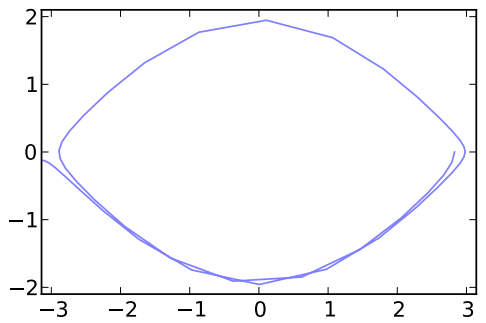

Figure 2: Phase diagrams for the damped pendulum problems integrated with Heun's method for various step sizes. Notice that the amount of dissipation (corresponding to the size of the hole in the middle) strongly depends on the step size. 


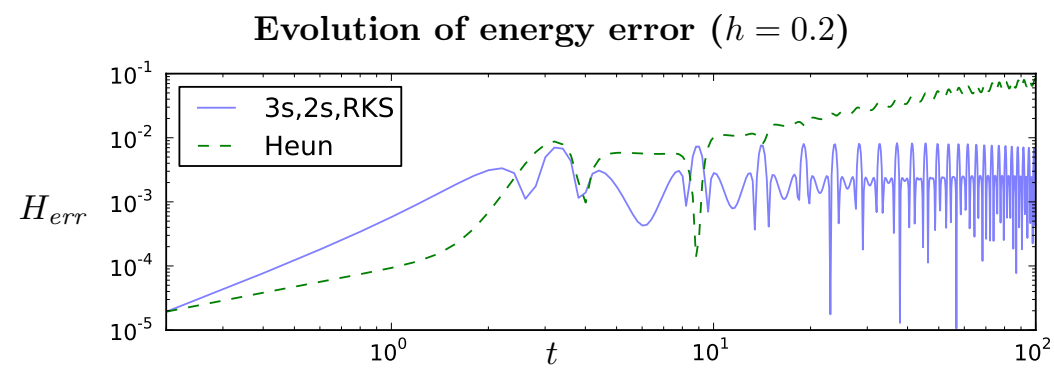

Comparison of energy in between methods $(h=0.2)$

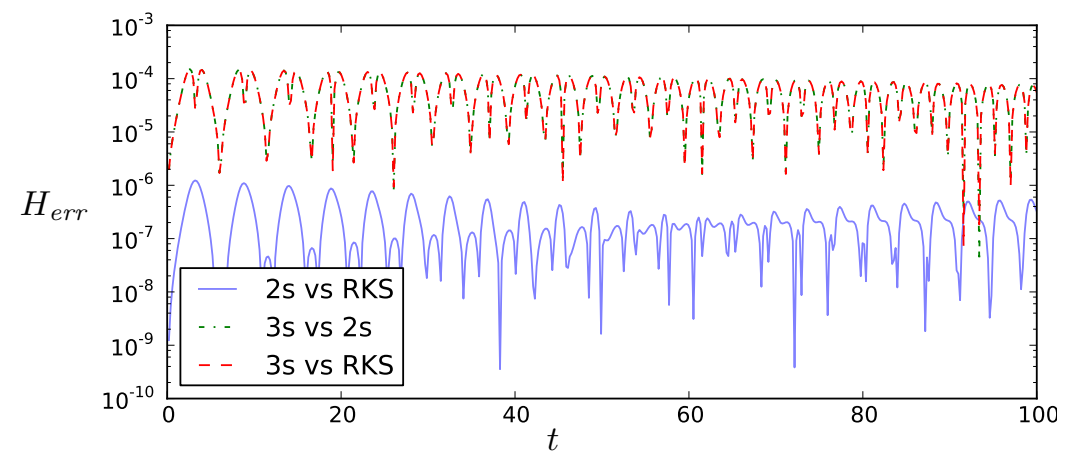

Figure 3: (Upper) Evolution of energy for Algorithms 3.1 3.3. and for Heun's method. The error is bounded for the suggested methods, whereas it grows linearly for Heun's method. (Lower) Comparison of energy evolution in between Algorithms 3.13 .3 The difference is about a factor $10^{2}$ or $10^{4}$ smaller than the actual energy error. 
unit mass and unit gravity (see e.g. 8, Sect. I.1] for details on this problem, in the un-damped case).

The system is integrated with each of Algorithm 3.1 3.3 as well as with Heun's explicit second order method. (We also use Heun's method as the choice of Runge-Kutta method for Algorithm 3.3. The following data was used: $q(0)=$ $0.9 \pi, p(0)=0, \varepsilon=10^{-2}$ and integration time interval [0,200], for various step sizes $h \in\left[10^{-1}, 1\right]$.

Phase diagrams for various step sizes using Algorithms $3.1 \sqrt{3.3}$ are given in Figure 1. There is virtually no difference between the suggested methods, i.e., the phase curves overlap. Notice that the size of the "hole" in the middle of the phase diagrams is almost independent of the step size. This verifies the result in Theorem 4.3 , that the dissipation rate is asymptotically correct independent of the step size. The corresponding phase diagrams for Heun's method (sometimes also called Runge's method) ${ }^{1}$ are shown in Figure 2 Notice that the dissipation rate in all the simulations is to small, and depends heavily on the step size. This reflects the fact that Heun's method (as most explicit Runge-Kutta methods) assembles "numerical" energy.

A detailed plot of the energy error as a function of time, is shown in the upper graph of Figure 3. (The "correct" energy is computed by highly accurate numerical integration.) In particular, notice that the energy error for the suggested methods seems to stay bounded, whereas it grows linearly for Heun's method. Although this result is not fully explained by our backward error analysis, it is related to the result obtained in Theorem 4.3 and Theorem 4.6, that the dissipation rate for the modified Hamiltonian is asymptotically correct in $\varepsilon$.

The lower graph in Figure 3 contains comparison of the energy between the suggested methods, i.e.,

$$
\left|H \circ \Phi_{2 \mathrm{~S}}^{h}-H \circ \Phi_{\mathrm{RKS}}^{h}\right|,\left|H \circ \Phi_{3 \mathrm{~S}}^{h}-H \circ \Phi_{2 \mathrm{~S}}^{h}\right|,\left|H \circ \Phi_{3 \mathrm{~S}}^{h}-H \circ \Phi_{\mathrm{RKS}}^{h}\right|
$$

(this difference is too detailed to recognize in the upper graph). Comparing with the values in the upper graph, notice that the difference in computed energy between the methods is much smaller than the actual energy error, i.e., they produce practically the same result. Also, notice that among the methods, the difference in result between $\Phi_{2 \mathrm{~S}}^{h}$ and $\Phi_{\mathrm{RKS}}^{h}$ is indistinguishable as compared to $\Phi_{3 \mathrm{~S}}^{h}$, i.e., $\Phi_{3 \mathrm{~S}}^{h}$ is the "out-lier" of the three.

\section{Symmetry and Conservation of Momentum}

In this section we show that the methods suggested in Section 3 preserve invariance under a symmetry, and conserve corresponding momentum maps. For preliminaries on geometric mechanics, see [17] or [1].

Let $\mathscr{G}$ be a Lie group acting on $\mathscr{Q}$ by $\phi: \mathscr{G} \times \mathscr{Q} \rightarrow \mathscr{Q}$. We write $\phi_{g}:=\phi(g, \cdot)$. Further, for $g \in \mathscr{G}$ we use the notation $g \cdot q=\phi_{g}(q)$ and $g \cdot z=\phi_{* g}(z):=$ $\left(T_{g \cdot q}^{*} \phi_{g^{-1}}\right)(z)$ for the lifted action. The action is associated with a corresponding momentum map $\mathrm{J}: T^{*} \mathscr{Q} \rightarrow \mathfrak{g}^{*}$, where $\mathfrak{g}^{*}$ is the dual of the Lie algebra $\mathfrak{g}$ of $\mathscr{G}$. Explicitly, the momentum map is defined by

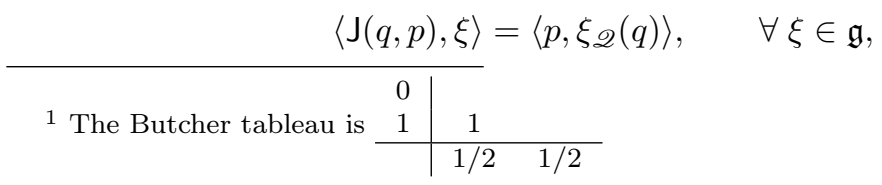


where $\xi_{\mathscr{Q}}$ is the infinitesimal generator of the action on $\mathscr{Q}$, i.e., $\xi_{\mathscr{Q}}(q):=$ $\left.\frac{\mathrm{d}}{\mathrm{d} t}\right|_{t=0}(\exp (t \xi) \cdot q)$.

Now, let $Y \in \mathfrak{X}\left(T^{*} \mathscr{Q}\right)$ and assume that its flow is $\mathscr{G}$-equivariant, i.e., that it commutes with the action:

$$
\exp (t Y) \circ \phi_{* g}=\phi_{* g} \circ \exp (t Y) .
$$

The infinitesimal version of this relation is $\left[Y, \xi_{T * \mathscr{Q}}\right]=0$ for all $\xi \in \mathfrak{g}$, where $\xi_{T^{*} \mathscr{Q}}(z):=\left.\frac{\mathrm{d}}{\mathrm{d} t}\right|_{t=0}(\exp (t \xi) \cdot z)$ is the infinitesimal generator of the action on $T^{*} \mathscr{Q}$. The vector field $Y$ is then called $\mathscr{G}$-invariant. Since the action on $T^{*} \mathscr{Q}$ is canonical, the vector field $\xi_{T^{*} \mathscr{Q}}$ is symplectic. In fact, it corresponds to the Hamiltonian function $J(\xi)=\langle\mathrm{J}(\cdot), \xi\rangle$, i.e., $\xi_{T * \mathscr{Q}}=X_{J(\xi)}$ so it is exact symplectic (see [17, Sect. 11.2]).

Associated with the momentum map is:

- The subgroup of $\mathscr{G}$-equivariant diffeomorphisms

$$
\operatorname{Diff}_{\mathscr{G}}\left(T^{*} \mathscr{Q}\right)=\left\{\varphi \in \operatorname{Diff}\left(T^{*} \mathscr{Q}\right) ; \varphi \circ \phi_{* g}=\phi_{* g} \circ \varphi, \forall g \in \mathscr{G}\right\} .
$$

Since $\xi_{T^{*} \mathscr{Q}}$ generates $\phi_{* \exp (t \xi)}$, the corresponding subalgebra of vector fields is given by

$$
\mathfrak{X}_{\mathscr{G}}\left(T^{*} \mathscr{Q}\right)=\left\{X \in \mathfrak{X}\left(T^{*} \mathscr{Q}\right) ;\left[X, \xi_{T^{*} \mathscr{Q}}\right]=0, \forall \xi \in \mathfrak{g}\right\} .
$$

- The subgroup of momentum conserving diffeomorphisms

$$
\operatorname{Diff}_{J}\left(T^{*} \mathscr{Q}\right)=\left\{\varphi \in \operatorname{Diff}\left(T^{*} \mathscr{Q}\right) ; J(\xi) \circ \varphi=J(\xi), \forall \xi \in \mathfrak{g}\right\},
$$

with corresponding subalgebra of vector fields given by

$$
\mathfrak{X}_{\mathcal{J}}\left(T^{*} \mathscr{Q}\right)=\left\{X \in \mathfrak{X}\left(T^{*} \mathscr{Q}\right) ; £_{X} J(\xi)=0, \forall \xi \in \mathfrak{g}\right\} .
$$

Notice that $\mathscr{G}$-equivariance does not necessarily imply conservation of the momentum map, not even in the Hamiltonian case ${ }^{2}$ Nor does conservation of momentum imply $\mathscr{G}$-equivariance. However, if a function $H \in \mathcal{F}\left(T^{*} \mathscr{Q}\right)$ is $\mathscr{G}$-invariant, i.e., $\{H, J(\xi)\}=0$ for all $\xi \in \mathfrak{g}$, then for the associated Hamiltonian vector field it holds that $X_{H} \in \mathfrak{X}_{\mathscr{G}}\left(T^{*} \mathscr{Q}\right) \cap \mathfrak{X}_{J}\left(T^{*} \mathscr{Q}\right)$ since $\left[X_{H}, \xi_{T^{*} \mathscr{Q}}\right]=$ $X_{\{H, J(\xi)\}}=0$ and $£_{X_{H}} J(\xi)=\{H, J(\xi)\}=0$.

Let $\mathfrak{X}_{\mathscr{G}, J}\left(T^{*} \mathscr{Q}\right):=\mathfrak{X}_{\mathscr{G}}\left(T^{*} \mathscr{Q}\right) \cap \mathfrak{X}_{J}\left(T^{*} \mathscr{Q}\right)$. We now give criterions for the vector fields involved in system (8) to be $\mathscr{G}$-invariant and/or momentum preserving.

Proposition 5.1. Let $\mathrm{g}, \mathrm{d}$ and $V$ be the tensors used in equation (4), and $X_{T}$, $Y$ and $X_{V}$ the corresponding vector fields in equation (8). Then,

$$
\begin{aligned}
& £_{\xi_{\mathscr{Q}}} \mathrm{g}=0 \quad \Longrightarrow \quad X_{T} \in \mathfrak{X}_{\mathscr{G}, \mathrm{J}}\left(T^{*} \mathscr{Q}\right) \\
& £_{\xi_{\mathscr{Q}}} V=0 \quad \Longrightarrow \quad X_{V} \in \mathfrak{X}_{\mathscr{G}, \mathrm{J}}\left(T^{*} \mathscr{Q}\right) \\
& \mathrm{i}_{\xi_{\mathscr{Q}}} \mathrm{d}=0 \quad \Longrightarrow \quad Y \in \mathfrak{X}_{\mathrm{J}}\left(T^{*} \mathscr{Q}\right) \\
& \left.\begin{array}{l}
£_{\xi_{\mathscr{Q}} \mathrm{g}}=0 \\
£_{\xi_{\mathscr{Q}}} \mathrm{d}=0
\end{array}\right\} \quad \Longrightarrow \quad Y \in \mathfrak{X}_{\mathscr{G}}\left(T^{*} \mathscr{Q}\right)
\end{aligned}
$$

\footnotetext{
${ }^{2}$ For example, take $\mathscr{Q}=\mathscr{G}=\mathbb{R}, H(q, p)=q$ and $J(\xi)(q, p)=p \cdot \xi$. Then $\left[X_{H}, \xi_{T^{*} \mathscr{Q}}\right]=$ $\left[X_{H}, X_{J(\xi)}\right]=X_{\{H, J(\xi)\}}=0$, but $£_{X_{H}} J(\xi)=\{H, J(\xi)\}=\xi \neq 0$.
} 
Proof. The result is already well known in the Hamiltonian case of $X_{T}$ and $X_{V}$. Indeed, $£_{\xi_{\mathscr{Q}}} \mathrm{g}=0$ implies that $\mathscr{G}$ acts on $T^{*} \mathscr{Q}$ by isometries. In turn, this implies that $T$ is $\mathscr{G}$-invariant. Further, $£_{\xi_{\mathscr{Q}}} V=0$ implies that $V$ is $\mathscr{G}$-invariant. In turn, $X_{T}$ and $X_{V}$ are $\mathscr{G}$-invariant and $\{T, J(\xi)\}=\{V, J(\xi)\}=0$. (See 24] or [1, Sect. 4.5] or [15, Chap. 3] for details).

The result remains to be shown for the non-Hamiltonian vector field $Y$. First the third implication. Since $Y(q, p)=\left(0,-\mathrm{d}_{q}\left(p^{\sharp}, \cdot\right)\right)$ and $J(\xi)(q, p)=\left\langle p, \xi_{\mathscr{Q}}(q)\right\rangle$, it holds that $£_{Y} J(\xi)(q, p)=-\mathrm{d}_{q}\left(p^{\sharp}, \xi_{\mathscr{Q}}(q)\right)$. Now, $\mathrm{d}_{q}\left(p^{\sharp}, \xi_{\mathscr{Q}}(q)\right)=\left(\mathrm{i}_{\xi_{\mathscr{Q}}} \mathrm{d}\right)_{q}\left(p^{\sharp}\right)=$ 0 by the presumption. Thus, $£_{Y} J(\xi)=0$, so $Y \in \mathfrak{X}_{J}\left(T^{*} \mathscr{Q}\right)$.

Next, the last implication. Let $\mathrm{f}$ denote the bi-linear form $T^{*} \mathscr{Q} \times T^{*} \mathscr{Q} \rightarrow \mathbb{R}$ given by $\mathrm{f}_{q}(p, r):=\mathrm{g}_{q}\left(p^{\sharp}, r^{\sharp}\right)=\left\langle p, r^{\sharp}\right\rangle$. If $£_{\xi_{\mathscr{Q}}} \mathrm{g}=0$ then $£_{\xi_{\mathscr{Q}}} \mathrm{f}=0$ (see [15, Sect. 3.1]). A calculation equivalent to the one in equation (6) yields

$$
\left(\phi_{* g}\right)_{*} Y(q, p)=\left(0,-\left(\phi_{* g} \mathrm{~d}\right)_{q}\left(\left(\phi_{* g} \mathrm{f}\right)_{q}(p, \cdot), \cdot\right)\right) .
$$

Chose now the path $g(t)=t \xi$, replace $g$ for $g(t)$, and take the time derivative $\left.\frac{\mathrm{d}}{\mathrm{d} t}\right|_{t=0}$. The left hand side then becomes the Lie derivative of $Y$ in the direction $\xi_{T * \mathscr{Q}}$. Using the product rule on the right hand side we get

$$
£_{\xi_{T^{*} \mathscr{Q}}} Y(q, p)=\left(0,-\left(£_{\xi_{\mathscr{Q}}} \mathrm{d}\right)_{q}\left(\mathrm{f}_{q}(p, \cdot), \cdot\right)-\mathrm{d}_{q}\left(\left(£_{\xi_{\mathscr{Q}}} \mathrm{f}\right)_{q}(p, \cdot), \cdot\right)\right) .
$$

By the presumption the right hand side vanishes, so $\left[\xi_{T^{*} \mathscr{Q}}, Y\right]=0$. Thus, $Y \in \mathfrak{X}_{\mathscr{G}}\left(T^{*} \mathscr{Q}\right)$.

We now have the following basic result, which concerns conservation of momentum and $\mathscr{G}$-invariance for the suggested methods.

Proposition 5.2. Let $\mathscr{H}$ denote any of $\operatorname{Diff}_{\mathscr{G}}\left(T^{*} \mathscr{Q}\right)$, $\operatorname{Diff}_{\lrcorner}\left(T^{*} \mathscr{Q}\right)$ or $\operatorname{Diff}_{\mathscr{G}, J} T^{*} \mathscr{Q}$, and let $\mathfrak{h}$ denote the corresponding subalgebra of vector fields. Then $X_{T}, Y, X_{V} \in$ $\mathfrak{h}$ implies that $\Phi_{3 S}^{h}, \Phi_{2 S}^{h}$ and $\Phi_{R K S}^{h}$, defined by Algorithms 3.1 3.3, belong to $\mathscr{H}$.

Proof. The result is clear for Algorithm 3.1 3.2 since $\mathscr{H}$ is closed under composition. Moreover, for $\Phi_{\mathrm{RKS}}^{h}=\exp \left(h X_{T} / 2\right) \circ \Psi_{X_{V}+\varepsilon Y}^{h} \circ \exp \left(h X_{T} / 2\right)$, it holds that the $\exp \left(h X_{T} / 2\right) \in \mathscr{H}$ if $X_{T} \in \mathfrak{h}$. The vector field $Y$ defines a linear system on $T_{q}^{*} \mathscr{Q}$. Since the momentum map is linear in $p$, and since all RungeKutta methods conserve linear invariants, it holds that $\Psi_{X_{V}+\varepsilon Y}^{h} \in \operatorname{Diff}_{\jmath}\left(T^{*} \mathscr{Q}\right)$ if $X_{V}, Y \in \mathfrak{X}_{\jmath}\left(T^{*} \mathscr{Q}\right)$. Furthermore, we notice that the lifted action $\phi_{* g}$ is exactly the co-tangent lift of $\phi_{g^{-1}}$. Thus, by Proposition 3.1 $\Phi_{\mathrm{RKS}}^{h}$ is invariant under the change of coordinates $(q, p)=\phi_{* g}(s, r)$. That is,

$$
\exp \left(h\left(\phi_{* g}\right)_{*} X_{T} / 2\right) \circ \Psi_{\left(\phi_{* g}\right)_{*}\left(X_{V}+\varepsilon Y\right)}^{h} \circ \exp \left(h\left(\phi_{* g}\right)_{*} X_{T} / 2\right) \circ \phi_{* g}=\phi_{* g} \circ \Phi_{\mathrm{RKS}}^{h} .
$$

Now, if $X_{T}, Y, X_{V} \in \mathfrak{X}_{\mathscr{G}}\left(T^{*} \mathscr{Q}\right)$, then $\left(\phi_{* g}\right)_{*} X_{T}=X_{T},\left(\phi_{* g}\right)_{*} Y=Y$ and $\left(\phi_{* g}\right)_{*} X_{V}=X_{V}$, so $\Phi_{\mathrm{RKS}}^{h} \circ \phi_{* g}=\phi_{* g} \circ \Phi_{\mathrm{RKS}}^{h}$, i.e., $\Phi_{\mathrm{RKS}}^{h} \in \operatorname{Diff}_{\mathscr{G}}\left(T^{*} \mathscr{Q}\right)$.

In particular, if $\mathrm{g}, \mathrm{d}$ and $V$ fulfill the requirements of Proposition 5.1, then it follows by Proposition 5.2 that Algorithms 3.13 .3 are both $\mathscr{G}$-equivariant and momentum conserving. It is important to point out that this result does not hold for general exact symplectic integrators, not even if the integrator is momentum preserving when $\varepsilon=0$. For example the alternative splitting method

$$
\Phi_{\text {RKS-B }}^{h}=\exp \left(h X_{V} / 2\right) \circ \Psi_{X_{T}+\varepsilon Y}^{h} \circ \exp \left(h X_{V} / 2\right)
$$

reduces to Störmer-Verlet when $\varepsilon=0$, but does not conserve momentum. 


\section{$6 \quad$ Near Conservation of Relative Equilibria}

In this section we investigate how the methods suggested in Section 3 preserve relative equilibria. For background on geometric reduction theory see e.g. [15. Chap. 3], [1, Chap. 4], or [16, Chap. 1-2].

Let $\mathscr{G}$ be a Lie group acting on $\mathscr{Q}$ as in the previous section. We assume that $\mathscr{G}$ acts freely and properly on $\mathscr{G}$ (cf. [15, Chap. 3]), which roughly speaking means that the action is non-singular.

Definition 6.1. Let $X \in \mathfrak{X}\left(T^{*} \mathscr{Q}\right)$. A solution curve $\gamma(t)$ to $\dot{z}=X(z)$ is called a relative equilibrium if there exists a $\xi \in \mathfrak{g}$ such that $\gamma(t)=\exp (t \xi) \cdot \gamma(0)$.

The study of relative equilibria of Hamiltonian systems on a co-tangent bundle $T^{*} \mathscr{Q}$ is closely related to the theory of co-tangent bundle reduction. The theme is to "quotient away" the part of the dynamics generated by the symmetry group $\mathscr{G}$, and thus, for each $\mu \in \mathfrak{g}^{*}$, obtain a reduced phase space given by $\mathscr{P}_{\mu}=\mathrm{J}^{-1}(\mu) / G_{\mu}$, where $\mathscr{G}_{\mu}$ is the subgroup of $\mathscr{G}$ that leaves $\mu$ invariant under the co-adjoint action, i.e., $\mathscr{G}_{\mu}=\left\{g \in \mathscr{G} ; \operatorname{Ad}_{g^{-1}}^{*} \mu=\mu\right\}$.

Remark 6.1. If $\mathscr{G}$ is an Abelian Lie group (all elements commute), it holds that $\mathscr{G}_{\mu}=\mathscr{G}$, so $\mathscr{P}_{\mu}=\mathrm{J}^{-1}(\mu) / \mathscr{G}$, and $\operatorname{dim} \mathscr{P}_{\mu}=2 \operatorname{dim} \mathscr{Q}-2 \operatorname{dim} \mathscr{G}$. In this case, the reduced phase space $\mathscr{P}_{\mu}$ is isomorphic to $T^{*}(\mathscr{Q} / \mathscr{G})$, equipped with a non-canonical symplectic structure $\omega_{\mu}$, depending on the momentum map value $\mu$. It holds that $\omega_{0}$ is the canonical symplectic structure on $T^{*}(\mathscr{Q} / \mathscr{G})$. In general, $\omega_{\mu}=\omega_{0}+\beta_{\mu}$, for a 2 -form $\beta_{\mu}$ usually called magnetic term. In classical mechanical examples, where $\mathscr{G}$ typically is a rotation group, $\beta_{\mu}$ corresponds to centrifugal forces due to the rotation. If $\operatorname{dim} \mathscr{G}=\operatorname{dim} \mathscr{Q}$ we have $n$ first integrals in involutions, i.e., the system is Arnold-Liouville integrable. The modern notion of co-tangent reduction in the Abelian case was developed by Smale 24]. However, its roots goes back to Lagrange, Poisson, Jacobi, and Routh 3

Let $\iota_{\mu}: \mathrm{J}^{-1}(\mu) \rightarrow T^{*} \mathscr{Q}$ be the natural inclusion, and $\pi_{\mu}: \mathrm{J}^{-1}(\mu) \rightarrow \mathscr{P}_{\mu}$ the projection $\pi_{\mu}(z)=[z]$, where $[z] \in \mathscr{P}_{\mu}=\mathrm{J}^{-1}(\mu) / \mathscr{G}_{\mu}$ is the equivalence class of $z \in \mathrm{J}^{-1}(\mu)$. Given $X \in \mathfrak{X}_{\mathscr{G}, \mathrm{J}}\left(T^{*} \mathscr{Q}\right)$ and initial data on $\mathrm{J}^{-1}(\mu)$, the flow $\exp (t X)$ restricts to the invariant momentum manifold $\mathrm{J}^{-1}(\mu)$. Further, due to the symmetry, it drops to a flow $\exp (t \bar{X})$ on $\operatorname{Diff}\left(\mathscr{P}_{\mu}\right)$ that fulfills $\pi_{\mu} \circ \exp (t X)=$ $\exp (t \bar{X}) \circ \pi_{\mu}$, where $\bar{X}$ is the vector field on $\mathscr{P}_{\mu}$ defined by $T \pi_{\mu} \circ X=\bar{X} \circ \pi_{\mu}$. Notice that for these constructions to make sense, it is essential that $X$ is both $\mathscr{G}$-invariant and momentum conserving. If $\gamma(t)$ is a relative equilibrium, then $\pi_{\mu} \circ \gamma(t):=[\gamma(t)]=[\gamma(0)]$. Indeed, $\gamma(t)$ is a relative equilibrium solution of the dynamical system $\dot{z}=X(z)$ if and only if $[\gamma(0)]$ is an equilibrium of $[\dot{z}]=\bar{X}([z])$, i.e., $\bar{X}([\gamma(0)])=0$.

Note that if $\gamma(t)=(q(t), p(t))$ is a relative equilibrium for system (8), then it must hold that $\mathrm{d}_{q(t)}\left(p(t)^{\sharp}, p(t)^{\sharp}\right)=0$ for all $t$, since the motion $\gamma(t)$ conserves the Hamiltonian.

Now, fix some $\varepsilon>0$ and let $Z=X_{T}+X_{V}+\varepsilon Y$ be the vector field for the system (8), and assume that g, d, $V$ fulfill all the requirements in Proposition 5.1 . so that $Z \in \mathfrak{X}_{\mathscr{G}, \mathrm{J}}\left(T^{*} \mathscr{Q}\right)$. Further, assume that system (8) has an asymptotically

\footnotetext{
${ }^{3}$ A historical account of the theory of co-tangent bundle reduction is presented in the introduction of the monograph by Marsden et. al. [16].
} 
stable relative equilibrium $\gamma(t)$, i.e., the critical point $[\gamma(0)]$ of $\bar{Z}$ is asymptotically stable. In general, an integrator $\Phi^{h}$ for $Z$ does not have a nearby relative equilibrium, not even for very small step sizes. The reason for this is that $Z$ is not structurally stable with respect to arbitrary perturbations in $\mathfrak{X}\left(T^{*} \mathscr{Q}\right)$. That is, for a small perturbation $\epsilon Z^{\Delta} \in \mathfrak{X}\left(T^{*} \mathscr{Q}\right)$ the vector field $Z+\epsilon Z^{\Delta}$ does not, in general, possess a perturbed relative equilibrium. However, the stationary point $[\gamma(0)]$ of the reduced vector field $\bar{Z}$ is structurally stable with respect to perturbations in $\mathfrak{X}\left(\mathscr{P}_{\mu}\right)$. That is, if $\epsilon \bar{Z}^{\Delta} \in \mathfrak{X}\left(\mathscr{P}_{\mu}\right)$, then for small enough $\epsilon$, the perturbed reduced vector field $\bar{Z}+\epsilon \bar{Z}^{\Delta}$ has a nearby stationary point. As a consequence, the relative equilibrium $\gamma(t)$ of $Z$ is structurally stable with respect to perturbations $\epsilon Z^{\Delta} \in \mathfrak{X}_{\mathscr{G}, J}\left(T^{*} \mathscr{Q}\right)$, since then the vector field $Z+\epsilon Z^{\Delta}$ drops to $\bar{Z}+\epsilon \bar{Z}^{\Delta}$ under symmetry reduction. The corresponding argument also works on the level of flow maps. Indeed, let $\Phi^{h} \in \operatorname{Diff} \mathscr{G}, \mathrm{J}\left(T^{*} \mathscr{Q}\right)$ be a $\mathscr{G}$-equivariant and momentum preserving integrator, approximating the exact flow $\exp (h Z)$. By the quotient map, the integrator drops to a map $\bar{\Phi}^{h} \in \operatorname{Diff}\left(\mathscr{P}_{\mu}\right)$, which approximates $\exp (h \bar{Z}) \in \operatorname{Diff}\left(\mathscr{P}_{\mu}\right)$, and since the fix point $[\gamma(0)]$ of $\exp (h \bar{Z})$ is hyperbolic, the perturbed map $\bar{\Phi}^{h}$ has a nearby fix point $\left[\gamma_{h}(0)\right]$ for $h$ small enough. In summary, we have the following:

Proposition 6.1. Let $\mathrm{g}, \mathrm{d}, V$ fulfill all the requirements in Proposition 5.1, and let $\Phi^{h}$ be a $\mathscr{G}$-equivariant and momentum preserving integrator for system 8 . Assume that $\gamma(t)$ is an asymptotically stable relative equilibrium. Then $\Phi^{h}$ preserves a nearby relative equilibrium for small enough step sizes.

Notice that the above result heavily relies on hyperbolicity, which in turn requires that the dissipation parameter $\varepsilon$ is strictly positive and the reduced dissipation tensor $\bar{d}$ is non-degenerate, since an equilibrium of a Hamiltonian system (typically) is elliptic. Thus, the requirement is that the step size is small enough in relation to the perturbation $\varepsilon Y$, i.e., essentially that $h^{r} \ll \varepsilon$.

Based on Theorem 4.6 we now derive a refined result, which states that Algorithm 3.1 preserves a stable nearby relative equilibrium for exponentially long times, independent of $\varepsilon$. Recall that a relative equilibrium $\gamma(t)$ is stable if it corresponds to some local minimum $[\gamma(0)]$ of the reduced Hamiltonian $\bar{H}$ (that is, $\mathrm{d} \bar{H}([\gamma(0)])=0$ and the Hessian of $\bar{H}$ at $[\gamma(0)]$ is strictly positive definite).

Theorem 6.2. Let $\mathrm{g}, \mathrm{d}, V$ be real analytic and fulfill all the requirements in Proposition 5.1. Assume that $\gamma(t)$ is a relative equilibrium of system (8), such that the critical point $[\gamma(0)]$ of $\bar{Z}$ is stable, and let $\overline{\mathrm{U}}$ be any compact neighborhood of $[\gamma(0)]$ such that the reduced Hamiltonian $\bar{H}$ is strictly convex on $\bar{U}$. Then there exists another neighborhood $\overline{\mathrm{V}} \subset \overline{\mathrm{U}}$ of $[\gamma(0)]$ and constants $h_{0}, \kappa, K>$ 0 , independent of $\varepsilon$, such that the reduced numerical solution $\left[z_{0}\right],\left[z_{1}\right], \ldots,\left[z_{n}\right]$ generated by Algorithm 3.1 stays in $\overline{\mathrm{U}}$ over exponentially long times $n h \leq K \mathrm{e}^{\kappa / h}$ whenever $\left[z_{0}\right] \in \overline{\mathrm{V}}, h \leq h_{0}$ and $\varepsilon \geq 0$.

Proof. Since the integrator $\Phi_{3 \mathrm{~S}}^{h}$ defined by Algorithm 3.1 is $\mathscr{G}$-equivariant and momentum preserving, it drops to a reduced integrator $\bar{\Phi}_{3 \mathrm{~S}} h \in \operatorname{Diff}\left(\mathscr{P}_{\mu}\right)$. Further, there is a reduced modified Hamiltonian given by $\bar{H}_{h, N}([z]):=H_{h, n}(z)$ for any $z \in[z]$ (well defined since $H_{h, N}$ is $\mathscr{G}$-invariant). Since $\bar{H}$ is strictly convex on $\mathrm{U}$, we may chose $h_{0}^{\prime}>0$ such that $\bar{H}_{h, N}$ is also strictly convex on 
$\overline{\mathrm{U}}$ whenever $h \leq h_{0}^{\prime}$, thus attaining a minimum $c_{\min }=\bar{H}_{h, N}\left(\left[z_{h, N}^{*}\right]\right)$ in the interior of $\overline{\mathrm{U}}$. Next, let $c_{\max }=\inf _{[z] \in \partial \overline{\mathrm{U}}} \bar{H}_{h, N}([z])$. It holds that $c_{\max }>c_{\min }$. Now, let $c_{\text {mid }}=\left(c_{\max }-c_{\min }\right) / 2$. It holds that the set $\overline{\mathrm{V}}=\{[z] \in \overline{\mathrm{U}}$ : $\left.\bar{H}_{h, N}([z]) \leq c_{\text {mid }}\right\}$ is a neighborhood of $\left[z_{h, N}^{*}\right]$ contained in the interior of $\overline{\mathrm{U}}$. Let $\left[z_{0}\right] \in \overline{\mathrm{V}}$. By Theorem 4.6 there exists $h_{0}^{\prime \prime}, \gamma, C, \lambda>0$ independent of $\varepsilon$ such that $H_{h, N}\left(\left[\Phi_{3 \mathrm{~S}}^{h}(z)\right]\right)-H_{h, N}([z]) \leq h C \mathrm{e}^{-\gamma / h}$ whenever $[z] \in \overline{\mathrm{U}}$ and $h \leq \min \left(h_{0}^{\prime}, h_{0}^{\prime \prime}\right)$, which implies that $H_{h, N}\left(\left[z_{n}\right]\right)-H_{h, N}\left(\left[z_{0}\right]\right) \leq n h C \mathrm{e}^{-\gamma / h}$. Since $\left[z_{0}\right] \in \overline{\mathrm{V}}$ it holds that $H_{h, N}\left(\left[z_{n}\right]\right) \leq c_{\max }$ whenever $n h C \mathrm{e}^{-\gamma / h} \leq c_{\max }-c_{\text {mid }}$, which yields the result since $H_{h, N}\left(\left[z_{n}\right]\right) \leq c_{\max }$ implies that $\left[z_{n}\right] \in \overline{\mathrm{U}}$.

The result states that if $\gamma(t)$ is a stable relative equilibrium solution to system 8 , then, for small enough step sizes, the numerical solution generated by Algorithm 3.1 will "almost" (for exponentially long times) preserve a modified relative equilibrium which is close to the exact one. In contrast to Proposition 6.1, where the analysis is based on structural stability of hyperbolic critical points, the step size restriction in Theorem 6.2 is independent of $\varepsilon$. In particular, the result holds in the conservative case when $\varepsilon=0$. Furthermore, no non-degeneracy assumption on the dissipation tensor $d$ is made: it may be degenerate in any direction.

\subsection{Relation to Symplectic Integration of Problems with Attracting Invariant Tori}

As mentioned in the introduction, there exists already a well developed theory for symplectic integration of Arnold-Liouville integrable systems, perturbed in such a way that only one invariant torus persist, and becomes attractive. For a thorough treatment of this theory, see [8, Chap. XII]. In this section we give a short review of that framework, and compare it to the setting and analysis presented in the current paper. The two approaches are related, but not overlapping.

Consider a perturbation of an integrable Hamiltonian system, which in action-angle variables (cf. [8, Chap. X]) can be written

$$
\begin{aligned}
\dot{\boldsymbol{a}} & =\varepsilon \boldsymbol{r}(\boldsymbol{a}, \boldsymbol{\theta}) \\
\dot{\boldsymbol{\theta}} & =\boldsymbol{\omega}(\boldsymbol{a})+\varepsilon \boldsymbol{\rho}(\boldsymbol{a}, \boldsymbol{\theta})
\end{aligned} \quad \boldsymbol{a} \in \mathbb{R}^{n}, \boldsymbol{\theta} \in \mathbb{T}^{n} .
$$

Further, assume there is a point $\boldsymbol{a}^{*}$ such that the frequencies $\boldsymbol{\omega}\left(\boldsymbol{a}^{*}\right)$ are diophantine with exponent $\nu$ (cf. [8, Chap. X]), and such that the angular average $\overline{\boldsymbol{r}}\left(\boldsymbol{a}^{*}\right):=\int_{\mathbb{T}^{n}} \boldsymbol{r}\left(\boldsymbol{a}^{*}, \boldsymbol{\theta}\right) \mathrm{d} \boldsymbol{\theta}$ is small and its Jacobian $A=\overline{\boldsymbol{r}}^{\prime}\left(\boldsymbol{a}^{*}\right)$ has strictly negative real part. Then system 15 has an invariant torus which attracts a neighborhood of $\left\{\boldsymbol{a}^{*}\right\} \times \mathbb{T}^{n}$ with exponential rate proportional to $\varepsilon$. Now, 8, Theorem 5.2, Chap. XII] states that if a symplectic integrator is applied to system (15) expressed in canonical variables $(\boldsymbol{q}, \boldsymbol{p}) \in \mathbb{R}^{2 n}$, then the numerical solution has a modified attractive invariant torus as long as $h^{r} \leq c_{0}|\log \varepsilon|^{-\kappa}$, where $\kappa=\max (\nu+n+1, r)$ and $c_{0}$ is a constant independent of $h, \varepsilon$.

Although Arnold-Liouville integrable systems are related to relative equilibria, in the sense that the former is a special case of the latter (see Remark 6.1), systems of the form (15) are only overlapping with systems of the form (8) in the trivial case when the attractive manifold consists of only one single point 
(corresponding to an asymptotically stable equilibrium). Indeed, the setting in this paper allows for a degenerate dissipation tensor, but has the requirement that the perturbation vector field $\varepsilon Y$ fulfills $£_{Y} H \leq 0$ for the Hamiltonian $H$. In contrast, the setting in [8, Chap. XII] does not require that the perturbation is monotonely decreasing energy, but instead that $\overline{\boldsymbol{r}}$ in equation 15 is non-degenerate. As a consequence, in order for system (8) to overlap with system (15) it is needed that the perturbed vector field fulfills $£_{Y} H<0$, i.e., that the dissipation tensor $\mathrm{d}$ has full rank. In the common case of systems that have a locally minimal energy point in phase space, this means that the corresponding attractive invariant manifold is must be an asymptotically stable equilibrium point.

As an example, take the Van der Pol equation studied in [8, Chap. XII]. Here $T^{*} \mathscr{Q}=\mathbb{R}^{2}$, with the harmonic oscillator Hamiltonian $H(q, p)=p^{2} / 2+q^{2} / 2$, and the perturbation is described by the tensor $\mathrm{d}_{q}(u, v)=\left(1-q^{2}\right) u v$ (i.e., the perturbation is linear in $p$ just as in this paper). However, for this tensor it does not hold that $£_{Y} H \leq 0$, since the tensor switches signature at $q^{2}=$ 1. This is indeed the very reason that the Van der Pol system has a nontrivial attractive invariant manifold: the requirement $£_{Y} H<0$ would force the invariant manifold to be the equilibrium point $(q, p)=(0,0)$. Likewise, the example considered in Section 6.2 below does not fit the setting in [8, Chap. XII], since the unperturbed system is not integrable, and since the dissipation tensor is degenerate.

In conclusion, one can say that the main differences between the type of systems analyzed in [8, Chap. XII] versus the ones in this paper, is the form of the perturbations, and that the former analysis requires the unperturbed system to be near integrable, whereas the latter requires the unperturbed system to be of standard form.

Furthermore, the results of the actual numerical analysis is of different character. This paper gives results on asymptotically correct dissipation rate (Theorem 4.3 and Theorem 4.6), and on near preservation of stable relative equilibrium for exponentially long times independent of the perturbation parameter $\varepsilon$ (Theorem 6.2). The analysis in [8, Chap. XII] gives results on near preservation of attractive invariant tori for indefinite time, but with step sizes depending weakly on $\varepsilon>0$. Also, as pointed out in [8, Chap. XII], any numerical integrator for system (15) preserve a nearby attractive manifold as long as $h^{r} \ll \varepsilon$. In contrast, for systems with relative equilibria the condition $h^{r} \ll \varepsilon$ is not enough in order for a general integrator to preserve nearby relative equilibria. Indeed, as derived in Proposition 6.1 above, the integrator should be $\mathscr{G}$-invariant and momentum preserving to assert that the numerical solution has nearby relative equilibria when $h^{r} \ll \varepsilon$.

\subsection{Numerical Example: Elastic 3-D Pendulum}

In this example we consider an elastic pendulum which is affected by gravity. The configuration space is $\mathscr{Q}=\mathbb{R}^{3} \backslash\{0\}$. There is a small Rayleigh damping term for the spring, but otherwise the pendulum is not damped. Thus, the dissipation is only active in the direction of the spring. The $\varepsilon$ parameter takes the rôle of the damping coefficient for the spring. Using Cartesian coordinates 
the equations of motion are:

$$
\left\{\begin{array}{l}
\dot{\boldsymbol{q}}=\frac{\boldsymbol{p}}{m} \\
\dot{\boldsymbol{p}}=-k\left(1-\frac{\ell}{|\boldsymbol{q}|}\right) \boldsymbol{q}+m \boldsymbol{g}-\varepsilon \frac{\boldsymbol{q} \boldsymbol{q}^{\top} \boldsymbol{p}}{m|\boldsymbol{q}|^{2}}
\end{array}\right.
$$

where $m$ is the mass, $\boldsymbol{g} \in \mathbb{R}^{3}$ is the gravity, $k$ is the stiffness of the spring, and $\ell$ is the length of the "unstretched" pendulum. Notice that the damping term only acts in the opposite direction of $\boldsymbol{q}$. Also, when $\boldsymbol{p}$ is perpendicular to $\boldsymbol{q}$ the damping vanishes. In terms of the presented framework we have

$$
\mathrm{g}_{q}(u, v)=\frac{m}{2} \boldsymbol{u}^{\top} \boldsymbol{v}, \quad \mathrm{d}_{q}(u, v)=\frac{\boldsymbol{u}^{\top} \boldsymbol{q} \boldsymbol{q}^{\top} \boldsymbol{v}}{|\boldsymbol{q}|^{2}}, \quad V(q)=\frac{k}{2}(\ell-|\boldsymbol{q}|)^{2}-\boldsymbol{q}^{\top} \boldsymbol{g} .
$$

Throughout the rest of the example, we work with unit constants: $m=k=\ell=$ 1 , and $\boldsymbol{g}=(0,0,-1)$.

Remark 6.2. For $\varepsilon=0$ the elastic pendulum problem is a non-integrable Hamiltonian system, meaning it has chaotic behavior; see [11, Chap. 5].

The problem has an $S^{1}$-symmetry by rotation about the $z$-axis. Indeed, the setting is $\mathscr{G}=S^{1}$ with action on $\mathscr{Q}$ generated by $\xi_{\mathscr{Q}}(\boldsymbol{q})=\xi \boldsymbol{e}_{3} \times \boldsymbol{q}$, where $\boldsymbol{e}_{3}=(0,0,1)$. It is straightforward to check that $\mathrm{g}, \mathrm{d}$ and $V$ fulfill all the conditions in Proposition 5.1. Thus, the flow evolves on $\operatorname{Diff}_{\mathscr{G}, \mathrm{J}}\left(T^{*} \mathscr{Q}\right)$. The corresponding conserved momentum map is the azimutal angular momentum given by $J_{3}(\boldsymbol{q}, \boldsymbol{p})=(\boldsymbol{q} \times \boldsymbol{p})_{3}$. In addition, it follows from Proposition 5.2 that Algorithms 3.13 .3 applied to this problem exactly conserves momentum.

The problem is integrated with Algorithms 3.1 3.3 and Heun's method, with the following initial data: $\boldsymbol{q}_{0}=(0,1.55884573,-0.6)$ and $\boldsymbol{p}_{0}=(1.34164079,0,0)$. The energy behavior for various step sizes and choice of $\varepsilon$ is shown in Figure 4 . Notice that the qualitative behavior of Algorithms 3.1 3.3, in terms of mean energy dissipation rate, is superior to the result with Heun's method. Indeed, contrary to Heun's method, the mean energy dissipation rate for the suggested methods does not depend much on the step size. Figure 5 shows the trajectory of the pendulum in the $x-y$-plane. Notice that that Heun's method does not "stabilize" around a limit cycle, which is the case for Algorithms 3.1 3.3. Instead, the trajectory drifts outwards in an exponentially increasing fashion. This is because Heun's method does not preserve a nearby relative equilibrium, whereas the analyzed methods do. We now continue with a discussion of relative equilibrium for the elastic pendulum.

Relative equilibria For each momentum value $\mu \in \mathfrak{g}=T_{\mathrm{Id}} S^{1}=\mathbb{R}$ there is a corresponding relative equilibrium for the elastic pendulum, corresponding to rotation with constant angular velocity about the vertical axis.

Turning to cylindrical coordinates $(\rho, \phi, z)$ on $\mathscr{Q}$, and corresponding cotangent lifted momenta $\left(p_{\rho}, p_{\phi}, p_{z}\right)$ on $T_{q}^{*} \mathscr{Q}$, the governing equations 16 take 
Energy diagrams for elastic pendulum
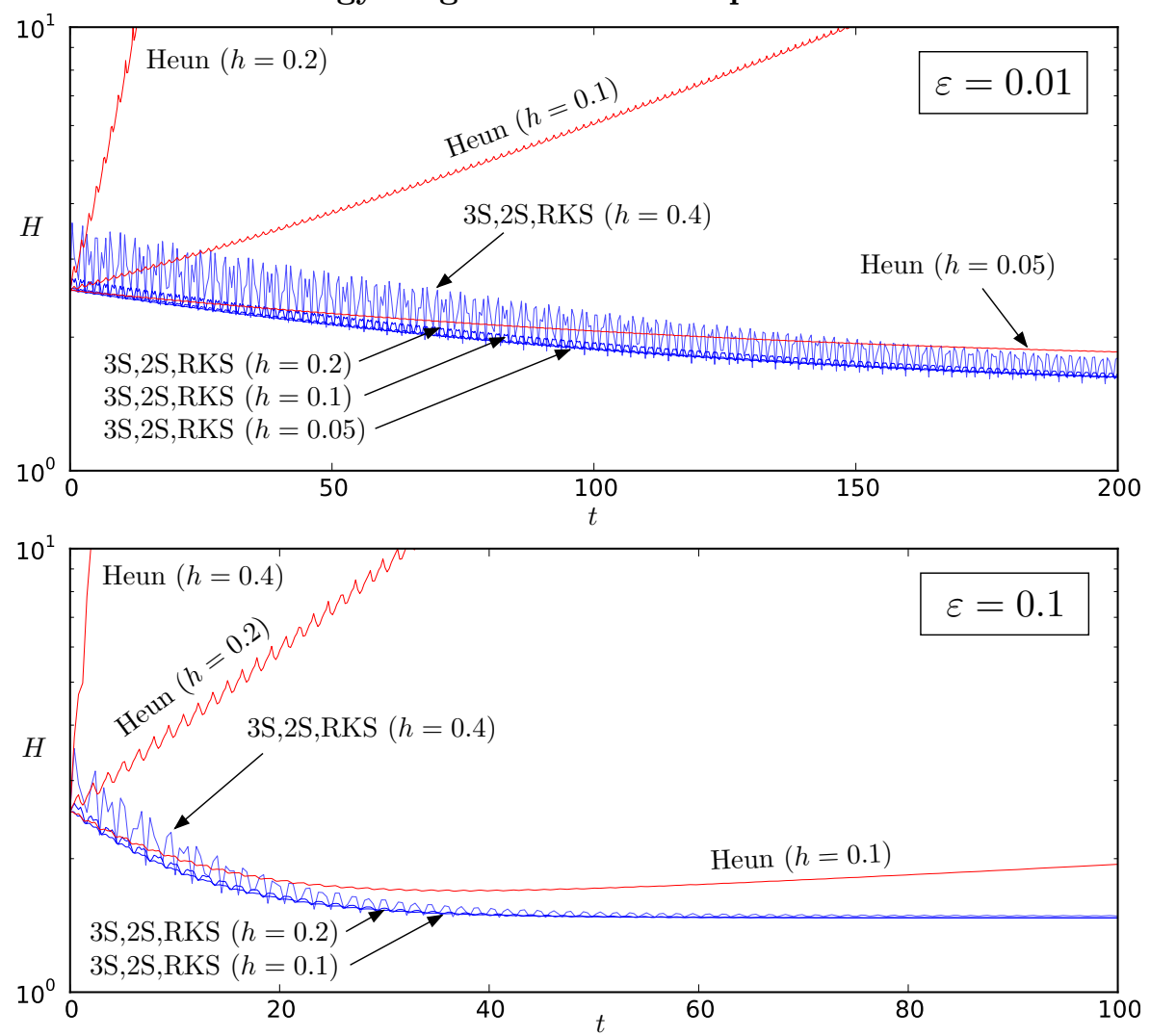

Figure 4: Energy diagrams for the elastic pendulum, using Algorithms 3.13 .3 and Heun's method, with $\varepsilon=0.01$ (upper) and $\varepsilon=0.1$ (lower). The energy oscillates for Algorithms 3.13 .3 but shows that qualitatively correct behavior, independently of the step size. For Heun's method, the result depends heavily on the step size, and is qualitatively incorrect, since energy is increasing. 
Projected $x-y$-trajectory for elastic pendulum

$3 \mathrm{~S}, 2 \mathrm{~S}, \mathrm{RKS}(h=0.1, \varepsilon=0.1)$

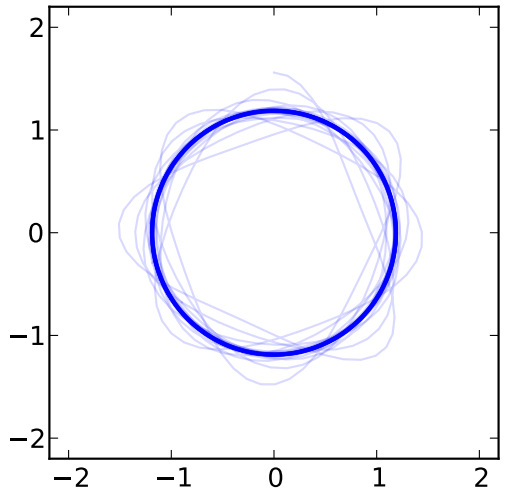

$3 \mathrm{~S}, 2 \mathrm{~S}, \mathrm{RKS}(h=0.1, \varepsilon=0.01)$

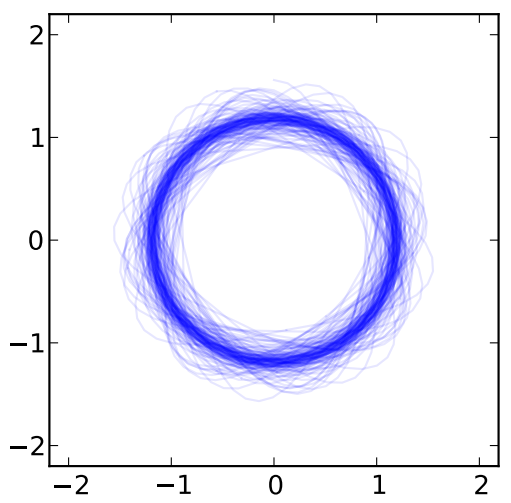

Heun $(h=0.1, \varepsilon=0.1)$

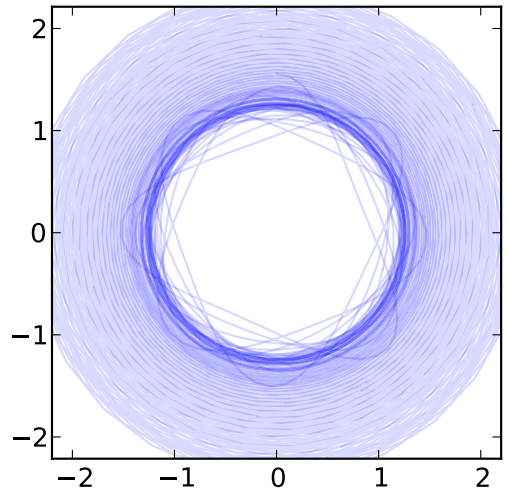

$3 \mathrm{~S}, 2 \mathrm{~S}, \mathrm{RKS}(h=0.4, \varepsilon=0.01)$

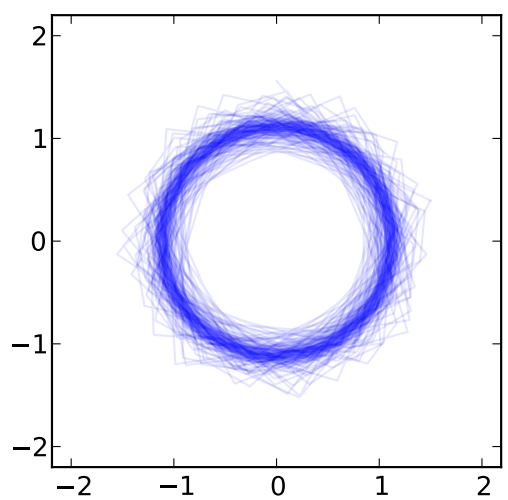

Figure 5: Trajectories in the $x-y$-plane for the elastic pendulum, using Algorithms 3.13 .3 and Heun's method. With Heun's method, the trajectory does not stay bounded, but drifts outwards. With Algorithms 3.1 3.3 the solution approaches a limit cycle (corresponding to a relative equilibrium). The exact correct limit cycle is not attained, but instead a modified one, in accordance with the analysis in Section 6 . 
Modified relative equilibria for Algorithms 3.13 .3

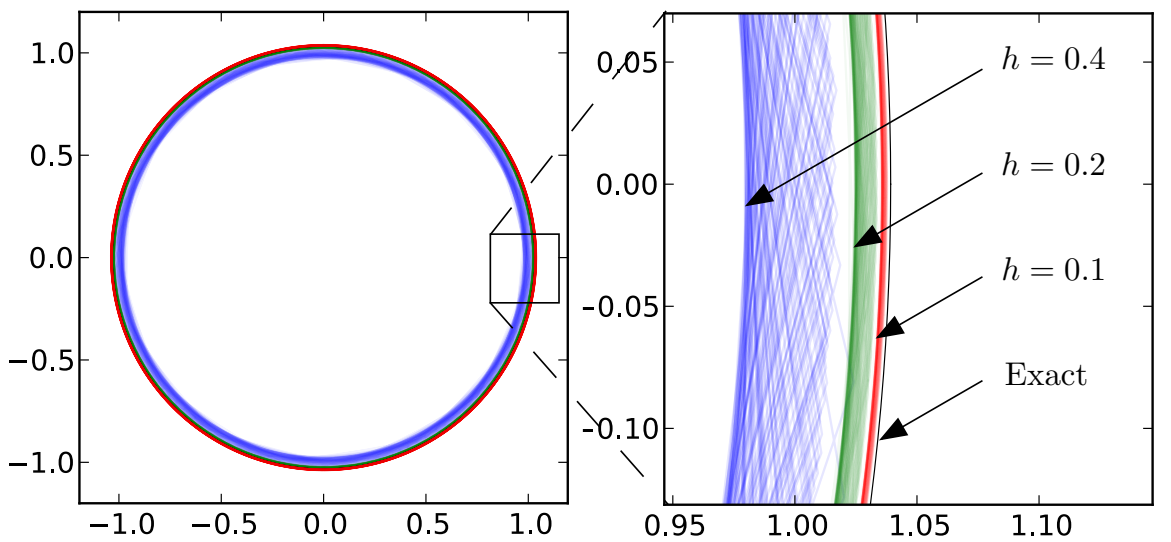

Figure 6: Near preservation of relative equilibria for Algorithms 3.1 3.3, with $\varepsilon=0.1$ (the results between the methods are inseparable). Initial data is given on the exact relative equilibrium cycle. The trajectory drifts towards a modified relative equilibrium cycle $\mathcal{O}\left(h^{2}\right)$ away from the exact cycle. The result verifies the analysis in Section 6 .

the form

$$
\begin{cases}\dot{\rho}=\frac{p_{\rho}}{m}, & \dot{p}_{\rho}=k \rho\left(\frac{\ell}{\sqrt{\rho^{2}+z^{2}}}-1\right)-\frac{p_{\phi}^{2}}{m \rho^{3}}-\varepsilon \rho \frac{z p_{z}+\rho p_{\rho}}{m\left(z^{2}+\rho^{2}\right)} \\ \dot{\phi}=\frac{p_{\phi}}{m \rho^{2}}, & \dot{p}_{\phi}=0 \\ \dot{z}=\frac{p_{z}}{m}, & \dot{p}_{z}=k z\left(\frac{\ell}{\sqrt{\rho^{2}+z^{2}}}-1\right)-m g-\varepsilon z \frac{z p_{z}+\rho p_{\rho}}{m\left(z^{2}+\rho^{2}\right)}\end{cases}
$$

Notice that $J_{3}(q, p)=p_{\phi}$. Due to the $S^{1}$-invariance of $\mathrm{g}, \mathrm{d}, V$, these equations reduce (in accordance with co-tangent reduction theory) to a system on $T\left(\mathscr{Q} / S^{1}\right)$. We may use the coordinates $\left(\rho, z, p_{\rho}, p_{z}\right)$, in which case the reduced equations are obtained by simply removing the equations for $\phi$ and $p_{\phi}$, and replacing $p_{\phi}$ with the momentum value $\mu$.

The equilibrium on the reduced space, corresponding to momentum value $\mu$, is given for initial data $\gamma(0)=\left(\rho, z, p_{\rho}, p_{z}\right)$ that fulfill the relations

$$
\begin{gathered}
k \rho\left(\frac{\ell}{\sqrt{\rho^{2}+z^{2}}}-1\right)-\frac{\mu^{2}}{m \rho^{3}}=0, \quad p_{\rho}=0 \\
k z\left(\frac{\ell}{\sqrt{\rho^{2}+z^{2}}}-1\right)-m g=0, \quad p_{z}=0 .
\end{gathered}
$$

Thus, the corresponding relative equilibrium solution $\gamma(t)=\exp (t \xi) \cdot \gamma(0)$ is given by

$$
\begin{cases}\rho(t)=\rho(0), & p_{\rho}(t)=0 \\ \phi(t)=\phi(0)+\frac{t \mu}{m(\rho(0))^{2}}, & p_{\phi}(t)=\mu \\ z(t)=z(0), & p_{z}(t)=0 .\end{cases}
$$


Since the action is given by $\exp (t \xi) \cdot(\rho, \phi, z)=(\rho, \phi+t \xi, z)$, it holds that $\xi=\mu /\left(m \rho(0)^{2}\right)$ for the equilibrium solution.

The $x-y$-trajectory using Algorithms 3.13 .3 and $\varepsilon=0.1$, with initial data

$$
\boldsymbol{q}_{0}=(0,1.0392304845413263,-0.6), \quad \boldsymbol{p}_{0}=(1.3416407864998736,0,0),
$$

corresponding to exact relative equilibrium for momentum value $\mu=p_{x}$, is given in Figure 6. Again, the result between the methods is inseparable. Notice that the trajectories slightly drift off the true relative equilibrium, toward a modified relative equilibrium. The rank of $d$ is one, meaning that the dissipation is degenerate even after the reduction (it is zero if $(z, \rho)$ and $\left(p_{z}, p_{\rho}\right)$ are orthogonal). Thus, we cannot apply Proposition 6.1 to explain the stability of the modified relative equilibrium for Algorithm 3.2 3.3 . However, notice that the reduced Hamiltonian is strictly convex in a neighborhood of the critical point $\left[\gamma_{h}(0)\right]$. Thus, by Theorem 6.2, the behavior seen in Figure 4 is fully explained for Algorithm 3.1 .

\section{Conclusions}

For Hamiltonian problems on standard form perturbed by Rayleigh damping, three numerical integration schemes were analyzed. When the dissipation parameter is zero, all the methods reduce to the Störmer-Verlet scheme. Based on backward error analysis, we gave result on:

- Asymptotically correct dissipation rate of the modified Hamiltonian (Theorem 4.3 and Theorem 4.6.

- Monotone decay of the modified Hamiltonian for small enough step sizes (Theorem 4.6).

- Preservation of symmetries, and conservation of momentum (Proposition 5.2.

- Near preservation of relative equilibria (Theorem 6.2).

The theoretical results were verified by numerical examples of a damped planar pendulum, and a damped elastic 3-D pendulum.

\section{Acknowledgment}

The authors would like the thank Claus Führer, Christian Lubich, and Ignacio Romero for helpful and inspiring discussions during the work with this paper. Also, we would like to thank the reviewers for helpful suggestions. The work was supported in part by the Royal Physiographic Society in Lund, Hellmuth Hertz' foundation grant, and the Marsden Fund in New Zealand.

\section{References}

[1] R. Abraham and J. E. Marsden, Foundations of Mechanics, second ed., Benjamin/Cummings Publishing Co. Inc. Advanced Book Program, Reading, Mass., 1978. 
[2] F. Armero and I. Romero, On the formulation of high-frequency dissipative time-stepping algorithms for nonlinear dynamics. I. Low-order methods for two model problems and nonlinear elastodynamics, Comput. Methods Appl. Mech. Engrg. 190 (2001), 2603-2649.

[3] F. Armero and I. Romero, On the formulation of high-frequency dissipative time-stepping algorithms for nonlinear dynamics. II. Second-order methods, Comput. Methods Appl. Mech. Engrg. 190 (2001), 6783-6824.

[4] O. Gonzalez, Time integration and discrete Hamiltonian systems, J. Nonlinear Sci. 6 (1996), 449-467.

[5] O. Gonzalez and J. C. Simo, On the stability of symplectic and energymomentum algorithms for non-linear Hamiltonian systems with symmetry, Comput. Methods Appl. Mech. Engrg. 134 (1996), 197-222.

[6] J. Grabowski, Free subgroups of diffeomorphism groups, Fund. Math. 131 (1988), 103-121.

[7] E. Hairer, C. Lubich, and G. Wanner, Geometric numerical integration illustrated by the Störmer-Verlet method, Acta Numer. 12 (2003), 399-450.

[8] E. Hairer, C. Lubich, and G. Wanner, Geometric Numerical Integration, second ed., Springer Series in Computational Mathematics, vol. 31, Springer-Verlag, Berlin, 2006.

[9] A. C. Hansen, A theoretical framework for backward error analysis on manifolds, J. Geom. Mech. 3 (2011), 81-111.

[10] M. Hochbruck and A. Ostermann, Exponential integrators, Acta Numerica 19 (2010), 209-286.

[11] D. D. Holm, Geometric Mechanics. Part I, Imperial College Press, London, dynamics and symmetry, 2008.

[12] C. Kane, J. E. Marsden, M. Ortiz, and M. West, Variational integrators and the Newmark algorithm for conservative and dissipative mechanical systems, Internat. J. Numer. Methods Engrg. 49 (2000), 1295-1325.

[13] A. Kriegl and P. W. Michor, The Convenient Setting of Global Analysis, Mathematical Surveys and Monographs, vol. 53, American Mathematical Society, Providence, RI, 1997.

[14] C. Lubich, B. Walther, and B. Brügmann, Symplectic integration of postnewtonian equations of motion with spin, Phys. Rev. D 81 (2010), 104025.

[15] J. E. Marsden, Lectures on Mechanics, London Mathematical Society Lecture Note Series, vol. 174, Cambridge University Press, Cambridge, 1992.

[16] J. E. Marsden, G. Misiołek, J.-P. Ortega, M. Perlmutter, and T. S. Ratiu, Hamiltonian Reduction by Stages, Lecture Notes in Mathematics, vol. 1913, Springer, Berlin, 2007.

[17] J. E. Marsden and T. S. Ratiu, Introduction to Mechanics and Symmetry, second ed., Texts in Applied Mathematics, vol. 17, Springer-Verlag, New York, 1999. 
[18] R. McLachlan and M. Perlmutter, Conformal Hamiltonian systems, J. Geom. Phys. 39 (2001), 276-300.

[19] R. I. McLachlan and G. R. W. Quispel, Splitting methods, Acta Numer. 11 (2002), 341-434.

[20] R. I. McLachlan and G. R. W. Quispel, Geometric integrators for ODEs, J. Phys. A 39 (2006), 5251-5285.

[21] K. Modin, Adaptive Geometric Numerical Integration of Mechanical Systems, Ph.D. thesis, Lund University, 2009.

[22] K. Modin, M. Perlmutter, S. Marsland, and R. I. McLachlan, On EulerArnold equations and totally geodesic subgroups, J. Geom. Phys. 61 (2011), 1446-1461.

[23] S. Reich, Backward error analysis for numerical integrators, SIAM J. Numer. Anal. 36 (1999), 1549-1570 (electronic).

[24] S. Smale, Topology and mechanics. I, Invent. Math. 10 (1970), 305-331. 